\title{
Space, Atmospheric, and Terrestrial Radiation Environments
}

\author{
J. L. Barth, Senior Member, IEEE, C. S. Dyer, E. G. Stassinopoulos, Member, IEEE
}

\begin{abstract}
The progress on developing models of the radiation environment since the 1960 s is reviewed with emphasis on models that can be applied to predicting the performance of microelectronics used in spacecraft and instruments. Space, atmospheric, and ground environments are included. It is shown that models must be adapted continually to account for increased understanding of the dynamics of the radiation environment and the changes in microelectronics technology. The IEEE Nuclear and Space Radiation Effects Conference is a vital forum to report model progress to the radiation effects research community.
\end{abstract}

\section{INTRODUCTION}

Topics in radiation environment research cover a broad subject matter because radiation exists throughout the universe originating from many sources and with varying intensities. A survey of the proceedings from the Nuclear Space and Radiation Effects Conference (NSREC), the December Issue of the IEEE Transactions on Nuclear Science (TNS) and TNS Special Issues, shows that radiation environment topics at NSREC are predicated by 1) critical radiation "effects and mechanism issues, 2) new atmospheric and space radiation measurements, and 3 ) availability of resources to transition new findings from basic science research to methods for quantitative predictions of the environment. The survey also reveals that many of the environment papers presented at NSREC were from authors who do not use the proceedings from NSREC as their primary forum for publication and that the NSREC papers were heavily referenced with papers from science journals. This implies that, as new issues in radiation effects were identified, the NSREC community borrowed knowledge from ongoing work in the science research community.

Manuscript received March 21, 2003. This by the NASA NEPPIERC Project and the Defense Threat-Reduction Ageney:-

Janet L. Barth, E.G. Stassinopoulos are with the NASA Goddard Space Flight Center, Greenbelt, MD 20771.

C.S. Dyer is with QinetiQ, Farnborough, UK
The "environments" session is a relatively new feature at the NSREC. The Space and Terrestrial Environments session was not part of the NSREC program until 1999. From 1991 through 1997, the environment papers were in the Spacecraft and Effects session. In the 1970 s and 1980 s environment papers were placed in the session of the effects issue that the environment topic addressed.

\section{OVERVIEW OF THE RADIATION ENVIRONMENT}

First a brief overview of space, atmospheric, and ground radiation environments will be presented. More complete descriptions of the radiation environments can be found in References [1], [2], [3], [4], and [5].

\section{A. Space Radiation Environments}

The natural space radiation environment can be classified into two populations, 1) the particles trapped by planetary magnetospheres in "belts", including protons, electrons, and heavier ions and 2) transient particles which include protons and heavy ions of all of the elements of the periodic table. The transient radiation consists of galactic cosmic ray (GCR) particles and particles from solar events, such as, coronal mass ejections and flares. These two types of solar eruptions produce energetic protons, alpha particles, heavy ions, and electrons that are orders of magnitude higher in abundance than the background GCRs.

Table 1 lists the maximum energy of space radiation particles. The table shows that much of the environment is high energy; therefore, shielding is not effective for many radiation source environments. When modeling, particles are treated as isotropic and omnidirectional with the exception of plasma, low altitude trapped protons $(<500 \mathrm{~km})$, and cosmic radiation on the ground. 
TABle 1: MAXIMUM ENergies of PARTiCles

\section{I) Trapped Populations}

James Van Allen is credited with discovering the trapped proton and electron regions around the Earth. Fig. 1 is an artist's drawing, which shows the belt-like structure of these particle regions. The tilt of the Earth's magnetic pole from the geographic pole and the displacement of the magnetic field from the center cause a dip in the field over the South Atlantic Ocean, resulting in a bulge in the underside of the inner belt. This region $(\sim 300$ to $\sim 1200 \mathrm{~km})$, not shown in Fig. 1, is called the South Atlantic Anomaly (SAA). In spite of the SAA's reputation for plaguing spacecraft, the flux levels there are actually much lower than those at higher altitudes. The $E>30 \mathrm{MeV}$ proton fluxes peak at approximately $2,500 \mathrm{~km}$ altitude at the equator. The electrons are trapped into two regions, the inner and outer zones. The $\mathrm{E}>2 \mathrm{MeV}$ peak electron fluxes at the equator are at approximately $2,500 \mathrm{~km}$ altitude in the inner zone and at $20,000 \mathrm{~km}$ altitude in the outer zone. Heavy ions are also trapped in planetary magnetic fields. For most shielded spacecraft systems, the abundances of these ions at energies high enough to penetrate spacecraft materials are too low to be a dominant factor in single event effects rates.

The trapped particle levels and locations are highly dependent on particle energy, altitude, inclination, and the activity level of the sun and are highly dynamic. The slot region population, between the inner and outer zones $\left(2<\mathrm{L}^{*}<2.8\right)$, and the outer zone ( $\left.\mathrm{L}>2.8\right)$ population can increase above averages by several orders of magnitude due to changes in the magnetosphere induced by solar and magnetic storms. Fig. 2, a plot of measurements of trapped electrons over a 1-year period, shows changes in the extremely dynamic outer zone and the slot region filling periodically with storm electrons. Due to their complex distribution and dependence on long- and short-term solar variability, the trapped particle populations are difficult to model and forecast.

The minimum requirement for the existence of a planetary radiation belt is that the planet's magnetic dipole moment must be sufficiently great to arrest the flow of the solar wind before the particles reach the top of the atmosphere where the particles will lose their energy due to collisions. Venus, Mars, and possibly Pluto do not have magnetospheres and, therefore, cannot support particle trapping. The magnetic fields of some of the other planets are similar to the Earth's, however, they vary in strength. Mercury has a weak

\footnotetext{
* Dipole shell parameter, $\mathrm{L}$, and rings of constant magnetic field strength are the two coordinates used to map trapped particles. $L$ is most simply described as the value that marks the particle drift shells by their magnetic equatorial distance from the center of the Earth.
}

magnetic field so it is expected it has a trapped particle population proportionally lower than that of the Earth. Saturn, Uranus, and Neptune have magnetic fields with similar strength to that of the Earth but measurements indicate that the intensities of the trapped radiation environments of Saturn, and Uranus are much lower than the Earth's and do not pose serious problems to the design of spacecraft systems. On the other hand, Jupiter's enormous magnetic dipole (428,000 compared to 30,760 nanoTesla for Earth) can support an intense particle environment. Its magnetosphere is the largest object in the solar system. Measurements have shown that the radiation environment is considerably more intense than the Earth's and is more extensive, therefore, mission planning for spacecraft that will spend even short times in the trapping regions of Jupiter must include careful definitions of the radiation environment. The Probos probe showed that Mars has a radiation environment, however, it is due to the thin atmosphere of Mars, which allows interplanetary GCRs and solar particles to penetrate to the surface. Interaction of these particles with the atmosphere produces neutrons, which penetrate to the planetary surface and then reflect back.

\section{2) Transient Populations}

The GCR population is a continuously present, slowly varying population of ions from all elements of the periodic table. The levels of GCRs are modulated by the 11-year solar cycle with the peak GCR populations occurring at solar minimum. Superimposed on the GCR levels are unpredictable, sudden rises in the flux levels due solar energetic particles (SEPs) from solar storms. Galactic and solar particles have free access to spacecraft outside of the magnetosphere. Because the transient particles penetrate the Earth's magnetosphere, they can reach near-Earth orbiting spacecraft and are particularly hazardous to satellites in polar, highly elliptical, and geostationary (GEO) orbits. Fig. 3 shows measurements from the IMP-8 spacecraft, which illustrates variations in the levels of carbon, oxygen, and nitrogen ions from GCR and solar sources over a $2 \hat{0}$ year period. The slowly varying, low-level GCR background population is seen to be approximately anticorrelated with the sunspot number. This is because the GCRs originate outside of our solar system and must "fight" against the solar wind to reach us. Fig. 3 also shows the solar particle events seen as spikes superimposed on top of the GCR measurements. These are the sudden increases in particle populations due to coronal mass ejections and/or solar flares.

\section{B. Atmospheric Environments}

As cosmic ray and solar particles enter the top of the Earth's atmosphere, they are attenuated by interaction with nitrogen and oxygen atoms. The result is a "shower" (Fig. 4) of secondary particles and interactions created through the attenuation process. 
Products of the cosmic ray showers are protons, electrons, neutrons, heavy ions, muons, and pions. In terms of radiation effects in the atmosphere, the most important product of the cosmic ray showers is the neutrons. They are measurable at $330 \mathrm{~km}$ altitude, and their density increases with decreasing altitude until they reach a peak at about $20 \mathrm{~km}$. At altitudes less than $20 \mathrm{~km}$, the levels decrease to where, at the ground, the neutron density is $1 / 500$ of the peak flux. Our knowledge of neutron levels comes from balloon, aircraft, and ground based measurements. The energies of neutrons in the atmosphere reach energy levels of $100 \mathrm{~s}$ of MeV. Taber and Normand [6] give an overview of the neutron environment in the atmosphere.

\section{Radiation on Earth}

Both natural and man-made radiation are present on the Earth. Table 2 lists these sources with their annual dose equivalents in units of milliSievert. The sources that are most important in producing effects in microelectronics on the ground outside of nuclear facilities are terrestrial and cosmic rays, which induce single event effects. The radiation effects on materials and microelectronics in nuclear facilities present special radiation effects challenges.

Cosmic radiation on the ground is from the products of $6^{\text {th }}$ and $7^{\text {th }}$ generation interactions of GCR and solar particles in the atmosphere. The population has a high vertical directionality. Variations in the primary population intensities cause most of the variations observed in the secondary neutron and proton levels. Levels rise and fall in the same 11-year solar cycle that modulates the GCRs. Sea-level cosmic rays are reduced by $30 \%$ during solar active periods. However, during large solar particle events, ground level enhancements (GLEs) of the terrestrial cosmic rays can increase the levels by as much as $5000 \%^{\dagger}$ depending on location and the size of the event [7]. Fig. 5 shows the components of cosmic rays at New York City as a function of energy. Studies have shown that the variation in the neutron flux level is measurable when the altitude ranges from sea level to mountainous regions.

TABLE 2: ANNUAL DOSE FROM GROUND RADIATION IN MSV [8]

${ }^{1}$ Dust particles bearing radon daughters expose lung tissue to alpha radiation.

${ }^{2}$ Naturally occurring radioactive elements (primarily potassium-40) other than radon daughters that find their way into our bodies

\section{TRAPPED RADIATION BELTS}

Birkeland theorized the existence of radiation trapping in planetary magnetospheres in 1895 when he performed vacuum chamber experiments to study

\footnotetext{
† Observed at Leeds during the February 1956 solar particle event.
}

aurora. With Poincare he showed that charged particles spiraled around field lines and are repelled by strong fields. Later Stöermer continued work of Birkeland on aurora and made calculations that led to the theory that there was a belt-like area around the earth in which particles were reflected back and forth between the poles. However, it was felt that the magnetic field was not strong enough to hold the particles. In 1957 Singer proposed that ring current could be carried by lower energy particles injected by into trapped orbits by magnetic storms. A complete history of radiation belt science can be seen in Reference 9.

\section{A. The Earths' Van Allen Belts \\ 1) Pre-NSREC: 1957-1963}

The launch of the Sputnik Earth orbiter by the Russians on October 4, 1957 sparked intense interest in developing a US space program. In 1955 James Van Allen and several other American scientists had proposed the launch of a scientific satellite as part of research programs to be conducted during the International Geophysical Year (IGY) of 1957-1958. The success of Sputnik led to the approval of Van Allen's proposal for the Explorer I spacecraft. Van Allen's interest in getting instruments into space was to study cosmic rays and their origin. He designed a cosmic ray detector to measure the low background cosmic rays, which was launched on Explorer I in January 31, 1958 from Cape Canaveral, Florida. In analyzing the data from his instrument, Van Allen was puzzled by "zero" readings. At first he thought that the instrument had malfunctioned but later he realized that the instrument was being "flooded" with radiation measurements. Van Allen determined that his instrument was measuring intense radiation surrounding the Earth, and he announced his discovery on May 1, $1958[10,9]$ Most of the scientific instruments flown in space in the late 1950s and early 1960 s were designed to detect energetic protons and electrons. With the results, scientists gained a general understanding of the nearEarth radiation environment but found differences up to a factor of 10 when making quantitative comparisons between measurements.

It was also during the early 1960 s that spacecraft electronics were found to be unreliable. Problems from differential charging from the solar wind and from noisy data transmission to the Earth from soft fails were noted. These problems were largely dealt with by building redundancy into systems. As the first scientific satellites were being launched in the late 1950s and early 1960s, the USSR and US also detonated nuclear devices at altitudes above 200 kilometers. The most dramatic of these tests was the US Starfish detonation on July 9,1962 . Ten known satellites were lost because of radiation damage, some immediately after the explosion [11]. The Starfish explosion injected enough fission spectrum electrons with energies up to $7 \mathrm{MeV}$ to 
increase the fluxes in the inner Van Allen belt by at least a factor of 100 . Effects were observed out to 5 Earth radii. The Starfish electrons that became trapped (modeled by Teague and Stassinopoulos [12]) dominated the inner zone environment $(\sim 2.8$ Earth radii at the equator) for five years and were detectable for up to eight years in some regions.

The production of enhanced radiation levels by the Starfish explosion and others and the ensuing problem of shortened spacecraft lifetimes emphasized the need for a uniform, quantitative description of the trapped particle environment. Wilmot Hess of the National Aeronautics and Space Administration's (NASA's) Goddard Space Flight Center (GSFC) developed the first empirical models of the trapped radiation belts. Using data from several satellites, he began constructing quantitative radiation models for inner zone protons and electrons. These models were designated as P1, P2, etc. and E1, E2, etc. Starting in 1962 and continuing through the late 1960s, several series of satellites were launched with instruments designed to measure the effects of Starfish, providing a large volume of particle data. In late 1963, James Vette of Aerospace Corporation and later of NASA/GSFC was appointed to lead a trapped radiation environment modeling program jointly funded by NASA and the United States Air Force (USAF). At that time, there were several groups actively involved in trapped particle measurements, including Aerospace Corporation, Air Force Cambridge Research Laboratory (now Air Force Research Laboratory), Johns Hopkins/Applied Physics Laboratory, Bell Telephone Laboratories, GSFC, Lawrence Livermore Laboratory, Lockheed Missile and Space Corporation, the University of California at San Diego, and the University of lowa. Each organization agreed to make its measurements available to the modeling program.

\section{2) NSREC 1965 - J. I. Vette}

At the 1965 NSREC, James Vette of the Aerospace Corporation presented an invited paper [13]. The focus of his paper was on modeling the trapped radiation belts. He referenced 31 papers from various scientific meetings and journals (AGU, Journal of Geophysical Research, etc., see Reference 13) where measurements and modeling results had been presented. Vette reviewed the efforts to model the outer zone particles, stressing the difficulty of developing static maps of the dynamic electrons in that region. He compared the lifetimes of the outer particles (minutes) to those of the inner zone (years). As his paper demonstrates, the modulation of the electrons driven by the 27-day solar rotation period and the fluctuations connected with magnetic activity were known at that time. Rather than presenting maps of the dynamic outer zone, he gave "typical" integral spectra. It was known at the time that the protons in the outer zone are more stable, are more closely confined to the magnetic equator, and have a "soft spectrum". With respect to damage to spacecraft, he notes that outer zone protons will affect only unshielded devices, but that electron exposure during long missions will result in measurable effects.

Vette presented the $\mathrm{AE}-\mathrm{1}^{\ddagger}$ map of the inner zone electron model and the newly developed AP maps for protons with energies greater than $4 \mathrm{MeV}$. Fig. 6 shows the AP-3 map that he presented for energies greater than $50 \mathrm{MeV}$. He noted that the natural inner zone electron population was not well known before Starfish and that Starfish electrons dominated the population levels in regions below $\mathrm{L}$ of 1.8 . He also stated that, before Starfish, protons up to several 100 s of $\mathrm{MeV}$ dominated the inner zone. Interestingly, as an aside, Vette made reference to a then recent observation by McIlwain that a redistribution of protons at $>34 \mathrm{MeV}$ followed a large magnetic storm in the $\mathrm{L}=2-3$ region [14].

3) Trapped Particle Model Development, 1960s, 1970 s, 1980s

Eight trapped proton models, eight trapped electron models, and one Starfish decay model were released during the 27 years that the trapped radiation modeling program was operative. The trapped particle models that are most often used at this time are the AP-8 [15] for protons and the AE-8 [16] for electrons. The AP-8 model, released in 1976, was the culmination of a longterm effort to include all of the previous models under one common approach and to include all of the data after 1970. After 1977, the modeling budget was significantly reduced so a similar effort to consolidate the electron models into the AE-8 model was not completed until 1983. The formal documentation of that model was released in 1991.

The AP- 8 and AE- 8 models include data from 43 satellites, 55 sets of data from principal investigator instruments, and 1,630 channel-months of data. By the 1970s, scientific interest had shifted from trapped particles to the plasma regime to determine the physical mechanisms of particle energization and transport. As a result, the number of new data sets available for trapped radiation environment modeling was drastically reduced. It was not until the measurement of storm belts by the CRRES mission in 1991 that concerns were renewed about the ability to model the trapped radiation belts to sufficient accuracy for using modern microelectronics in space.

4) The STARFISH Exo-Atmospheric, HighAltitude Nuclear Weapon Test

In 1958 the United States conducted the HARDTACK series of nuclear weapons tests over the Pacific Ocean and the ARGUS series in the South Atlantic Ocean. In 1962 additional tests were conducted

\footnotetext{
† The " $A$ " is for Aerospace Corporation where Vette first worked.
} 
in the FISHBOWL series. One test, the STARFISH PRIME, with a yield of 1.4 megatons TNT equivalent, was exploded on July 9, 1962 at a very high altitude (approximately $400 \mathrm{~km}$ ) over Johnson Island in the Pacific Ocean (about 700 miles from Hawaii). This exoatmospheric nuclear explosion released about $10^{29}$ fission electrons into the magnetosphere, creating an artificial radiation belt and raising the intensity of the inner zone electron population by several orders of magnitude. This additional radiation increased the radiation damage on spacecraft flying in that region to critical levels. The first failure (due to total ionizing dose) was the TELSTAR satellite, which was launched one day after the STARFISH. It was estimated that the spacecraft experienced a total dose from the explosion that was 100 times larger than was planned for the spacecraft lifetime. Within months after the tests, seven satellites failed, primarily due to solar cell damage.

Initial predictions of the longevity of the STARFISH debris ranged from the overly optimistic of some months to the more realistic of a few years. Studies conducted in the late 1960 s $[17,18,19,20]$ attempted to define the rate of decay with varying results. An in-depth evaluation was performed in 19701971 [21] using data from the 1963-38C satellite that covered the time span from September 1963 to December 1968. The researchers identified three distinct regions within the inner zone domain that were populated by the artificial electrons and established that their decay lifetime $\tau$ in days could be best expressed by. a function of three variables:

\section{Region 1}

$$
\tau_{1}=\tau(B, L, E) \quad \text { in }
$$

Region 2

$$
\tau_{2}=\tau(L, E) \text { in }
$$

Region 3

$$
\tau_{3}=\tau(E) \quad \text { in }
$$

where $\mathrm{B}$ is the field strength in gauss, $\mathrm{L}$ is the magnetic shell parameter, and $\mathrm{E}$ is the energy in $\mathrm{MeV}$. Fig. ? shows the dependence of the decay lifetime on $L$ for $E=$ $0.28 \mathrm{MeV}$. In 1972 a more thorough approach produced a model of the STARFISH flux for September 1964 based on data from several spacecraft (OGO-1, OGO-3, OGO-5, OV3-3, and 1963-38C) [12]. That model distinguished between artificial and natural electrons and provided the artificial flux as a function of equatorial pitch angle, energy, and $L$ value. The decay times for this flux were determined by two separate methods, which were combined to yield average values that are appropriate for the evaluation of the long-term loss process of the artificials. A map of the threshold energy as a function of $L$ for decay cutoff times is shown in Fig. 8.
Numerical values relating to nuclear explosions must include a substantial margin of error. In addition to the difficulties in making measurements of these events at the time of their occurrence, the results are dependent on circumstances that cannot be predicted. Two nuclear weapons of different design may have the same explosive energy yield, but the effects could be markedly different. In the case of the STARFISH, estimates of errors associated with the cutoff-time model are given in Table 3.

TABLE 3: ACCURACY OF THE STARFISH DECAY MODEL IN MONTHS

It is interesting to compare the STARFISH effects with those of a Soviet high-altitude test of a low-yield weapon that was performed on October 28, 1962 over Semipalatinsk in Kazakhstan. Fig. 9 shows the integral Van Allen belt electron fluxes before and after the event in the regions form $L$ of $\sim 1.8-4.0$ for particles with energies of $E>0.5$ and $E>1.9 \mathrm{MeV}$. This region covers the outer edge of the inner zone, the slot region, and the beginning of the outer zone. The bulk of the fission electrons are concentrated between $L$ of $\sim 1.8$ 2.7 , which is to be expected because their injection occurred at a high latitude location. In contrast, the STARFISH debris was concentrated in the inner zone due to the low latitude of the Johnson test site. A schematic of the distribution of the fission electrons from these two tests is shown in Fig. 10 in terms of magnetic shell parameter $\mathrm{L}$ and magnetic latitude. Fig. 11 is an attempt to show the average lifetimes of the $\mathrm{E}>$ $2 \mathrm{MeV}$ electrons from the STARFISH and Soviet experiments. Although it is difficult to draw conclusions from only two tests, the data suggest that longevity is maximum at low $\mathrm{L}$ values, decreases rapidly towards the slot region, and is in the range of months and perhaps weeks at larger $L$ values.

\section{5) The Storm Belts - The CRRES Mission}

A discussion of developments in our knowledge of the trapped radiation environment cannot be complete without including the contributions that were made by the CRRES mission. Not only did the mission provide data for radiation belt modeling, but it also rekindled interest in radiation belt science. The CRRES satellite carried a full complement of particle detectors, and its geosynchronous transfer orbit of $350 \mathrm{~km}$ perigee and over $30,000 \mathrm{~km}$ apogee at $18^{\circ}$ inclination was ideal for measuring radiation belt particles. Unfortunately, the CRRES launch was too late to measure the large September and October 1989 solar events. However, CRRES was in operation for the second peak of solar cycle 22 and observed the largest magnetic event ever recorded in March of 1991. Analysis of data from the instruments on CRRES before and after the March 1991 magnetic storm showed extremely large redistributions of the trapped particle populations. At the 1991 NSREC, Mullen et al. [22] reported that the CRRES proton instrument had measured a new proton storm belt in the 
slot region $(L=2-3)$. In Fig. 12 this belt for $47 \mathrm{MeV}$ protons is seen as a region outside of the inner zone belt of protons. In the April 1996 Special Issue of the TNS, Gussenhoven et al. [23] summarized all of the CRRES results including a review of this proton belt formation and the production of an electron storm belt during the same storm. Recall from Section III.A that in 1965 McIlwain previously reported that he had observed a redistribution of protons at $\mathrm{E}>34 \mathrm{MeV}$ following a large magnetic storm in the $L=2-3$ region.

One of the most important conclusions that resulted from the CRRES program was that existing theory could not explain the particle penetrations deep into the magnetosphere observed by McIlwain and Mullen et al. In $1993 \mathrm{Li}$ et al. [24] used a simplified model of the Storm Sudden Commencement (SSC) compression of the magnetosphere to show that electron belts like those measured by CRRES can be created in tens of seconds when the interplanetary shock wave from the storm interacts with the magnetosphere. Later Hudson et al. [25] showed that this shock acceleration theory could also be used to explain the sudden formation of proton storm belts.

The frequency of occurrence of these atypical events is unknown, therefore, applying current models to setting design and operational rad-hard requirements creates uncertainties that are impossible to quantify. Dynamic environment simulations are a method that could be used to address this issue. In the April 1996 Special Issue of the TNS, Boucher et al. [26] reviewed modeling techniques that provide computer generated models of trapped particle transport during storms. Boucher and Bourdarie [27] are developing a 4-D diffusion code to calculate the transport of particles throughout the inner magnetosphere. They have applied the code to several problems, including calculation of the transport of existing and injected particles during storms, determination of the ring current growth using calculations for both protons and electrons, and discovery of the process by which high-energy particles are totally removed from the internal magnetosphere. Fig. 13 gives an example of the output from the code where the diffusion of $100 \mathrm{keV}$ electrons in the radiation belts is plotted after injection of particles at midnight while the field increases (simulating a storm). The differential fluxes are in units of electrons $\mathrm{MeV}^{-}$ ${ }^{2} \mathrm{~cm}^{-2} \mathrm{~s}^{-1}$. Case studies have been validated using CRRES and STRV-1b measurements [28]. In the future they plan to drive the model with interplanetary magnetic field strength thereby greatly increasing the database for running the code and providing statistics for a range of conditions for a given time period.

\section{6) European Space Agency (ESA) Model Improvements}

A paper in the April 1996 Special Issue of the TNS highlighted some of the work by the European Space
Agency (ESA) in improving the trapped radiation models. Daly et al. [29] identified errors in the NASA models and documentation, including a source code error in AP-8-MIN and the fact that the AZUR dataset on which the AP-8-MAX is based covered a time span of 3 months, not 6 months. They also noted that the Space Shuttle and the LDEF satellite measured environments in low altitudes ( 300 to $500 \mathrm{~km}$ ) were from 60 to $100 \%$ higher than those predicted by the AP8 models. The authors determined that a large source of this error is due to the method used to interpolate between the $\mathrm{B} / \mathrm{B}_{0}$ values in the regions near the atmospheric cutoff. They developed an alternate interpolation method that increased the estimated fluxes by 10 to $40 \%$ bringing them closer to the measured levels. They also recommend that an additional $L$ increment at the low $\mathrm{L}$ values be included in the models to give better resolution at steep gradients. Fig. 14 shows the steep gradients below $\mathrm{L}$ of 1.4. The additional $\mathrm{L}$ increment increased the estimated fluxes by about $40 \%$. When these two refinements to the interpolation scheme were combined, the revised flux levels were much closer to the measured values. Daly's work renewed interest in improving the radiation belt models for applications to enabling and commercial off the shelf technologies, which can have very low radiation tolerance.

7) Radiation Belt Models with Improved Time Resolution

Analysis of the CRRES instrument and experiment data showed that not only is the environment extremely dynamic but also that electronic parts respond to the short-term changes. The AP- 8 and AE- 8 radiation belt models, with their 4-6 year averages, were adequate for long mission durations and for long-term degradation effects. However, modern spacecraft and instrument systems have serious problems with short-term effects, such as, interference and data corruption. With a time resolution of 4-6 years, the radiation belt models place a serious restriction on the ability to address design and operation issues of systems.

Several researchers have recognized the need for trapped particle models with finer time resolution. Three empirical models were developed using CRRES data to estimate short-term dynamic changes in the particle population, the CRRESPRO [30], CRRESELE [31], and CRRESRAD [32]. The CRRESPRO gives estimates of the trapped proton levels before and after the March 1991, simulating quiet and active conditions in the magnetosphere. CRRESELE gives estimates for eight conditions of magnetic activity, six ranges of activity as determined by the $A_{P 15}$ magnetic index and for the average and worst case conditions that CRRES measured. The CRRESRAD model was developed from data measured by the CRRES 4-domed dosimeter and includes quiet and active conditions. While the models 
are based on data collected over a short 14-month period and during solar maximum conditions only, they give the most comprehensive picture available of the environment resulting from a geomagnetic storm. Reference 23 gives an overview of the models, including a comparison to the AP-8 and AE- 8 models and a description of their limitations. Later data from a similar dosimeter flown on the APEX satellite was used to extend the CRRESRAD model to low altitudes and high latitudes. The APEXRAD [33] dose model includes the contributions from outer zone electrons and their variability, which was investigated as a function of magnetic activity using the $A_{P_{15}}$ index.

Models with improved time resolution for the low altitude portion of the Earth's radiation belts have been developed in recent years. Pfitzer [34] plotted predicted flux values for low inclination orbits as a function of the average atmospheric density. From this index, he interpolated and extrapolated fluxes from the AP-8 and AE- 8 models for solar activity conditions. Later Huston and Pfitzer [35] analyzed proton instrument data from the CRRES and TIROS/NOAA satellites with the goal of developing a low altitude $(<850 \mathrm{~km})$ trapped proton model with variation over an entire solar cycle as a function of solar activity indices (Fig. 15). At NSREC 1998, Huston presented the first trapped proton model with true solar cycle variation [36]. The proton flux levels were determined by using the solar radio flux proxy for atmospheric heating and included the phase lag between rise and fall of flux levels and solar activity.

Recently, the Huston team joined efforts with Xapsos and others at GSFC to add statistical variations to the solar cycle driven model thereby adding confidence level information to proton levels. This increases the ability to address trapped proton variations for spacecraft design and mission planning. This work was presented at the 2002 NSREC [37]. The authors note that this model is still in development and requires further analysis and validation.

Heynderickx and Lamaire [38] also plotted model fluxes as a function of the average weighted density of the atmosphere (ns) that is encountered by a particle on its drift shell. They found that the relationship between ns and the AP-8 and AE-8 models fluxes is well ordered, especially at low $L$ values. If a practical form for the ns calculation is developed, this method could lead to replacing $\mathrm{B}_{0}$ with $\mathrm{B}_{0}(\mathrm{~ns})$ when accessing the AP8 and AE- 8 models, thereby reflecting solar activity effects. Using data from the proton instrument on the SAMPEX spacecraft, they also developed a trapped proton model with improved time resolution [39].

\section{B. Trapped Radiation at Other Planets}

I) NSREC 1971 - J.W. Haffner

After numerous missions to explore the Earth's radiation belts and interplanetary space, scientists became interested in exploring the outer planets. For the 1971 IEEE TNS, J. W. Haffner contributed a paper, "Natural Radiation Environments for the Grand Tour Missions" [40] that presented an overview of the solar "flare", galactic, and magnetically trapped particle radiation expected during missions to the four outer planets, Jupiter, Saturn, Uranus, and Pluto. He estimated the environment levels from data and models and predicted mission doses. The ability to predict the environments at the outer planets was greatly hampered by the lack of measurements of the interplanetary environment beyond 1 astronomical unit (AU) and by the lack of any measurements of the trapped radiation environments of Jupiter and Saturn.

Haffner gave estimates of the trapped radiation belts of Jupiter and Saturn. It was theorized that Jupiter and Saturn had belts similar to those of the Earth based on decametric (bursts) and decimetric (quasi-steady state) radio frequency radiation emitted by Jupiter and Saturn. Similar if radiation emission was measured from the Earth's belts. The decimetric radiation is due to synchrotron emission of the electrons trapped in the magnetic field, the decametric radiation is associated with one of Jupiter's moons, Io. Based on assumptions about the limiting particle fluxes, similar relationships in Jupiter's and Earth's magnetic fields, and particle and plasma densities, and by ignoring the effect of the planet itself, Haffner derived relationships between the magnetic field at Jupiter's equator, the particle density relative to the plasma stability limit, and the effective inner radius of the Jovian belts. From that set of parameters, the electron dose rates for mission flybys were estimated.

The same methodology could not be applied to trapped protons because they do not radiate as the electrons do. Haffner pointed out that theory explaining the source and loss mechanisms for protons or electrons in the Earth's belts that could be applied to estimating the proton belts of Jupiter did not exist. Therefore, he had to base the proton estimates for Jupiter and Saturn on the ratios of the protons/electrons in the Earth's Van Allen belts. He used these estimates to calculate doses for the missions.

\section{2) NSREC 1972 - Paul Kase}

At the 1972 NSREC Kase followed up Haffner's work with a presentation that focused on concerns about displacement damage on spacecraft electronics due to proton and neutron environments [41]. In addition to natural sources, Kase presented the problem posed by having neutron emitting radio-isotope thermoelectric generators (RTGs) on board spacecraft. Kase also 
revisited the problem of modeling the proton belts of Jupiter. By that time, Divine had developed nominal and upper limit models of the proton belts [42]. The three orders of magnitude difference between the two models was an indication of the inaccuracy inherent in the unvalidated theoretical approach. It was expected that the proton spectra were "very hard" near the surface of Jupiter and softer at great distances.

\section{3) Measurements of the Grand Tour Mission}

The Pioneer missions to the outer planets carried instruments to measure the radiation environment. The measurements showed that the radiation was orders of magnitude higher than expected. Peak intensities of electrons in the belts, as measured by Pioneer 10 , were 10,000 times greater than Earth's maximum. Also, the electron energies were found to be greater than $20 \mathrm{MeV}$. Protons were several thousand times as intense as Earth's belts. The inner radiation belts of Jupiter, as measured by Pioneer 10 , had the highest radiation intensity so far measured, comparable to radiation intensities following an explosion of a nuclear device in the upper atmosphere. Pioneer 11 confirmed these high intensities. In the inner region of the magnetosphere, protons exceeding $35 \mathrm{MeV}$ appear to peak in two shells; the outer shell was detected at 3.5 Jovian radii by Pioneer 10 and confirmed by Pioneer 11 , and an inner shell, discovered by Pioneer 11, has a peak at 1.78 radii of Jupiter. Pioneer 11 also found that there is a greater flux of energetic particles at high Jovian latitudes than would have been expected from the measurements made by Pioneer 10. It also discovered that the flux of energetic particles peaks on either side of the dipole magnetic equator [43]. This discovery led to the need to retrofit the Galileo spacecraft with radiation hardened bipolar processors, because the spacecraft design with unhardened processors had been fixed before the arrival of the Pioneer spacecraft at Jupiter [44]. The models of the Jupiter radiation environment were updated using data from the missions to the outer planets [45].

\section{SOlAR PRoton EVENT MOdEL DEVELOPMENT}

\section{A. Early Estimates of Solar Proton Levels}

At NSREC 1965 Vette compared the levels of solar "flare" protons and galactic cosmic rays based on work by Malitson and Webber [46] and noted that the solar protons are low during periods of low solar activity. He also pointed out that they were considered a serious hazard for astronauts on Apollo missions. To predict the solar proton environment, Haffner compiled all of the existing solar proton data at $1 \mathrm{AU}$ for 1956-1968, including data from Webber, McDonald, Lewis, Modisette, and Mosley (see Reference 46). He developed fitting functions to fill in missing data that described the onset, rise time, and decay of solar events as a function of time. He then reconstructed probable values based on sunspot numbers by using predictions of future sunspot numbers developed by Weddell and Haffner [47]. To estimate the diffusion of the solar protons throughout interplanetary space, Haffner assumed a spatial dependence of $1 / r^{2}$ where $r$ is measured in $A U$, which is an approximation still used today. The method was used to predict the expected solar particle environment for each year from 1970 to 1989. Haffner points out the uncertainties in predicting solar particle levels. In fact, as seen in Table 4, the differences between the solar proton levels predicted for 1972 and 1989 at $1 \mathrm{AU}$ and those measured by spacecraft are large.

\begin{tabular}{ccc} 
TABLE 4: & SOLAR PROTONS > $10 \mathrm{MEV}$ \\
\hline Year & $\begin{array}{l}\text { Predicted } \\
\mathrm{p} / \mathrm{cm}^{2} / \mathrm{yr}\end{array}$ & $\begin{array}{c}\text { Actual } \\
\mathrm{p} / \mathrm{cm}^{2} / \mathrm{yr}\end{array}$ \\
\hline 1972 & $7.0 \times 10^{9}$ & $2.4 \times 10^{10}$ \\
1989 & $8.0 \times 10^{8}$ & $4.5 \times 10^{10}$ \\
\hline
\end{tabular}

\section{B. The First Statistical Models}

As concern grew over electronics and human exposure to solar protons and as the amount of available on-orbit particle measurements increased, the use of compilations of data for estimating solar proton levels was replaced by modeling efforts. In 1974 King [48] published the first statistical model for solar proton events using Poisson distributions. He concluded from his analysis of proton data from the 20th solar cycle that solar proton events could be classified into "ordinary" and "anomalously large". This was based on the fact that only one anomalously large event occurred in the 20th solar cycle - the August 1972 event. That event alone accounted for $84 \%$ of the total proton fluence in the solar cycle at energies $E>30 \mathrm{MeV}$. Stassinopoulos [49] developed the solar proton model, SOLPRO, based on King's statistical analysis. However, when Feynman et al. [50] added cycle 19 and 21 data to the solar proton event database, they were able to conclude that individual solar proton events actually form a continuum of event severity from the smallest to the largest, blurring the distinction between ordinary and anomalously large events.

\section{Engineering Oriented Statistical Models}

Many large events similar to the August 1972 event occurred in cycle 22 increasing concern about the validity of the solar proton models. With the goal of improving the ability to address practical aspects of spacecraft reliability, a team led by Xapsos began compiling solar proton data for solar cycles 20,21 and 22 and using statistical techniques to derive probability distributions of cumulative solar proton fluences. A review of the data sets can be found in Reference 51. At 
the 1996 NSREC, Xapsos et al. [52] presented a paper that described the application of extreme value theory to determine probability of encountering a single large event over the course of a mission. They also used compound Poisson process theory to describe the probability of encountering various fluence levels during a mission. The work of the Xapsos team confirmed the Feyman conclusion that a "typical event" cannot be defined.

The Xapsos team then turned their focus to understanding how to define the peaks of solar proton events. To accomplish this, they applied Maximum Entropy Principle (MEP) to select the least biased event probability distribution. The MEP, used for earthquake predictions, is valuable for analyzing incomplete datasets. They validated the results with Lunar Rock Records dating back to ancient times. New peak values for $E>10 \mathrm{MeV}$ were published in the 1998 TNS [53]. The Xapsos team continued their work by establishing worst case solar proton spectra for solar events [54]. When comparing their model with the CREME96 [55] "worst-week" solar proton model, which was based on the October 1989 solar particle event, they found that, statistically, the CREME96 model is closer to a $90 \%$ worst case event model [56]. Xapsos et al. have combined the model elements into the Emission of Solar Protons (ESP) model, which is available as a computer code $[51,56]$. Fig. 16 compares energy spectra for the new ESP model with the King and Feynman models.

\section{HEAVY IONS}

\section{A. Heavy Ions Prior to NSREC 1975}

In the early 1900s, scientists were puzzled by "charge" on electroscope leaves while performing laboratory experiments. The leaves of an electroscope repelled each other without the presence of charge. Scientists assumed that this was the result of the ionization of air by the natural radiation present on Earth (see Table 3). Efforts were made to eliminate the radiation by using radiation pure materials, however, the problem persisted. In 1913 an Austrian scientist, Victor Hess, devised an experiment to put an electroscope in a balloon to get it away from the Earth's radiation. As Hess and his experiment ascended in the balloon, he observed that the radiation source did not go away rather, as the altitude of the balloon increased, the radiation increased. Hess concluded that the source of this radiation was from outer space. In the summer of 1925, Millikan confirmed with his lake experiments that the radiation source was indeed from outer space. When he presented his lake experiment findings, he called the radiation "cosmic rays" [57]. In 1936 Hess and C. D. Anderson received the Nobel Prize for their discovery of cosmic rays.

Intense interest in understanding cosmic rays continued into the 1950s. Recall from Section III.A that
Van Allen's interest in getting instruments into space was to study cosmic rays and their origin. The first discussion of GCRs at NSREC was in 1965 when Vette [13] compared the levels of solar "flare" protons and galactic cosmic rays based on work by Malitson and Webber [46]. The primary concern was dose levels on spacecraft components, but it was determined that, in the presence of protons and electrons, the dose from heavy ions is a non-issue.

In the late $1960 \mathrm{~s}$, the interest in cosmic rays of solar and galactic origin went beyond basic scientific research and became a safety issue when astronauts on Apollo missions reported visual light flashes. McNulty [58] proposed that Cerenkov radiation generated by individual cosmic ray ions traversing the vitreous of the eye were responsible for the flashes. $\mathrm{He}$ and his colleagues proceeded with a series of experiments that exposed human subjects, P. McNulty, V. P. Pease, V. P. Bond, and L. Pinsky, to energetic heavy ions at accelerators. Understanding of the source and mechanism of the light flashes raised the concern for astronaut and safety, which in turn generated interest in measuring and modeling the galactic and heavy ion space environment. In fact, the IMP-8 spacecraft, which has provided the best long-term data set of heavy ions, was planned as a result of these concerns.

Haffner discussed the concern for the GCR contribution to total dose on spacecraft in his 1971 NSREC paper [40]. He presented values for the levels of galactic cosmic rays ${ }^{\S}$ and estimated that the expected GCR dose for the Grand Tour missions was in the range of a few hundred rads.

\section{B. NSREC 1975 - New Concerns about Heavy lons}

Prior to NSREC 1975, heavy ion populations, whether of galactic or solar origin, were not considered a major concern for the reliability of spacecraft electronics. Regardless of the region of space that missions visited, the contribution of heavy ions to spacecraft charging, ionizing dose, or displacement damage effects were insignificant compared to other sources of radiation, such as, the trapped radiation belts or protons from solar events. That changed at NSREC 1975 when Binder et al. [59] reported, "Anomalies in communication satellite operation have been caused by the unexpected triggering of digital circuits. Although the majority of these events have been attributed to charge buildup from high temperature plasmas, some of the events appear to be caused by another mechanism." In addition to an analysis of the circuit effects and the basic mechanism of these events, the authors presented cosmic ray spectra of Meyer [60], and they calculated the intensities using abundances from various authors, including Burrell and Wright [61] who investigated

\footnotetext{
$\S$ The source of the data is not referenced.
} 
dose rates of GCRs for astronaut exposure. Previous research on galactic cosmic rays by magnetospheric physicists interested in basic scientific research and by nuclear physicists concerned with astronaut dose became significant for the NSREC community. As explained by McNulty [58], the interaction models used to explain the light flashes observed by astronauts eyes were modified slightly to explain the upsets observed in microelectronic circuits. The Binder paper sparked intense interest in modeling heavy ion environments and interactions. (see other papers in this volume)

C. The Cosmic Ray Effects on Microelectronics (CREME) Code

A team at Naval Research Laboratory, led by Jim Adams, recognized the need for a comprehensive software package to calculate single event upset (SEU) rates in space that integrated environment predictions with particle interaction models. They embarked on the task of developing a comprehensive computer tool that could be used by researchers and engineers. Due to the extent of the upset problem, the effort had to include compilation of data sets for GCR and solar heavy ion populations, development of GCR and solar heavy ion models, evaluation of solar proton data and models, development of magnetospheric cutoff calculations, analysis of spacecraft shielding effects, and development interaction models for upsets due to heavy ions and protons. Adams et al. produced two Naval Research Laboratory (NRL) Memorandum Reports, which reported on topics related to this task [62, 63]. The first report, published in 1981, contained a comprehensive review of the near-Earth particle environment.

\section{1) The CREME Heavy Ion Environment Models}

The GCR environment model was based on data from several researchers collected through 1980. (see Adams [62]). Because of the dissimilar shape of their energy spectra, the hydrogen, helium, and iron ion distributions were treated as separate cases, and the other elements were scaled to one of the three spectra, as appropriate, using the relative abundances of the elements. Four different models of the GCR environment were developed, one of them being the well known " $90 \%$ worst case environment".

The solar heavy ion environment was more difficult to model because of the unavailability of a good dataset from spacecraft instrumentation. Adams et al. assumed that the solar particle events with the highest proton fluxes are always heavy ion rich and estimated fluence levels for the higher energy solar heavy ions $(>1 \mathrm{MeV})$ by scaling the abundances to protons. Eight different models of the solar heavy ion environment were developed because, without the benefit of comprehensive space measurements, the authors had to account for all possible solar activity conditions.
2) Extending the Models to a Rate Prediction Tool-CREME86

At NSREC 1982 Adams et al. presented a paper [64] that, with a paper by Petersen et al. [65], essentially laid the groundwork for the CREME86 code. This code was the first end-to-end desktop capability to calculate radiation environments throughout near-Earth regions and to use laboratory test parameters from devices to calculate the rate of a radiation effect. The simple title, "The Natural Radiation Environment Inside Spacecraft," does not reflect the complexity and groundbreaking nature of the work. The paper reviewed the work on the development of the environment models [62] and presented methods to calculate the transport of particles through the magnetosphere and through spacecraft shielding. The authors also showed the utility of using linear energy transfer (LET) spectra to represent the heavy ion environment in a form that condenses the energy spectra of all ions into a compact expression and that can be applied to calculating energy transfer in microvolumes. Heinrich constructed the first LET spectrum describing the ion environment in space in 1977 for biomedical purposes [66, see also 67]. At 1978 NSREC, Pickel and Blanford showed the applicability of the LET spectrum to the single event upset problem in microelectronics [68]. The resulting CREME [69] code was first released in 1986.

\section{3) Updates to the CREME Code}

For ten years the CREME86 code was a standard for calculating heavy ion environments. As knowledge of the radiation environment increased, it became apparent that the CREME86 models could be improved. Using ISEE 3 data, Reames et al. [70] found an inverse correlation between proton intensity and the iron/carbon heavy ion abundance ratio and that the composition of a solar particle event was a result of the location of the event on the sun. This contradicted Adams' assumption that all solar events are He rich, meaning that the solar heavy ion models were probably over predicting. Dyer et al. [54] measured LET spectra during the March 1991 event with the CREDO instrument on UoSAT-3. When they compared the measurements with LET calculated using the CREME86 solar particle models, they found that, in the LET range important for single event effects analyses, all of the models severely overpredicted the LET levels. Dyer et al. [71] measured LET spectra during several events, including the March 1991 event, with the CREDO instrument on UOSAT-3. When they compared the measurements with the LET spectra calculated using the CREME86 solar particle models, they found that, in the LET range important for single events from ions (greater than $0.5 \mathrm{MeVmg}^{-1} \mathrm{~cm}^{2}$ ), all of the models severely overpredicted the fluxes.

In the 1990 s NRL recognized the need to improve the environment models in the CREME86 code. The most important update to the code was the solar heavy 
ion model. Dietrich from the University of Chicago analyzed the solar heavy ion data from the IMP-8 satellite, providing the most comprehensive set of solar heavy ion space data to date [72]. The dataset is especially important for modeling the fluences at higher energies. A team led by Tylka used the results to model the solar heavy ions based on the October 1989 solar particle event. An analysis of 100 solar heavy ion events in the Dietrich database showed that this event could be used as a representative of a "worst case" environment. Tylka et al. presented the CREME96 model the 1997 NSREC [55]. The CREME96 solar heavy ion estimates are significantly lower than the heavy ion models in CREME86. Fig. 17 compares the LET energy spectra for the CREME86 and CREME96 solar heavy ion models. Recently Dyer et al. [73,74] have presented data on the LET spectra of solar particle events occurring between 1998 and 2001. While for many of the events there are very low enhancements of high LET fluxes, three events do approach or equal the CREME96 worst day model. At low LET, where protons dominate and usually lead to single event effects by nuclear interactions, two events slightly exceed the model. This is consistent with the suggestion by Xapsos et al. (see Section IV.C) that the CREME96 model is a $90 \%$ worst case. They are applying the methodology used in the ESP solar proton model to the solar heavy ion measurements to derive a statistically based solar heavy ion model.

The GCR environment model was also updated to include the analysis of the SAMPEX measurements of anomalous cosmic rays (ACRs). With the finding that the ACRs are not singly charged over energies of 20 $\mathrm{MeVn}^{-1}$, four models in CREME86 were replaced with one GCR model in CREME96. The model change significantly reduced the calculated single event effects rates for high threshold devices in low altitude orbits [55].

\section{ATMOSPHERIC AND GROUND ENVIRONMENTS}

\section{A. Atmospheric Environments}

In the last twenty years it has been discovered that electronics in aircraft systems, which are subjected to increasing levels of cosmic radiation and their secondaries as altitude increases, are also sensitive to single event effects. At the 1984 NSREC Tsao et al. [75] presented methods for calculating LET spectra and SEU rates from cosmic rays and their secondary fragments propagating down to 40,000 feet. Later the calculation of this component of the atmospheric radiation environment was embodied in the Air Propagation (AIRPROP) code [76]. In a companion paper to the Tsao et al. paper, Silberberg et al. [77] presented methods for calculating the SEU rates arising from the secondary neutrons generated by the interactions of cosmic rays with the atmosphere. These papers demonstrated that the ions are important at greater than about 65,000 feet but that SEUs from neutron interactions dominate at lower altitudes. They also predicted that SEU rates would increase during large solar particle events.

An increasing body of data on upsets in avionics systems were accumulated that pointed to neutrons as the being the primary cause of SEUs on aircraft. In an unintentional experiment in 1993, reported by Olsen et al. [78], a commercial computer was temporarily withdrawn from service when bit-errors were found to accumulate in 256 Kbit CMOS SRAMs (D43256 A6U$15 \mathrm{LL})$. Following ground irradiations by neutrons, the observed upset rate of $4.8 \times 10^{-8}$ upsets per bit-day at conventional altitudes (35,000 feet) was found to be explicable in terms of SEUs induced by atmospheric neutrons. In an intentional investigation of single event upsets in avionics, Taber and Normand [79] flew a large quantity of CMOS SRAM devices at conventional altitudes on a Boeing E-3/AWACS aircraft and at high altitudes (65,000 feet) on a NASA ER-2 aircraft. Upset rates in the IMS1601 64Kx1 SRAMs varied between $1.2 \times 10^{-7}$ per bit-day at 30,000 feet and $40^{\circ}$ latitude to $5.4 \times 10^{-7}$ at high altitudes and latitudes. Reasonable agreement was obtained with predictions based on neutron fluxes.

Our knowledge of neutron levels comes from balloon, aircraft, and ground based measurements. These studies show that the energies of the neutron flux range from $\mathrm{keV}$ to hundreds of $\mathrm{MeV}$. For SEU calculations in aircraft systems, it is usually only the energies greater than $10 \mathrm{MeV}$ that are significant. It is also known that the flux peaks at an altitude of about 60,000 feet, which is the same altitude of the peak of observed SEU rates. Because the shape of the neutron spectrum varies little over altitude, models can be greatly simplified. However it has recently been demonstrated by Dyer and Lei [80] that aircraft structures, passengers and fuel can produce significant thermal neutron fluxes, leading to potentially high rates of SEU in components containing the nuclide boron-10 in borophosphosilicate glass passivation layers.

Two coordinate systems are commonly used to define the neutron distributions, energy-altitude-latitude and energy-atmospheric depth-magnetic rigidity. Taber and Normand [6] have developed an empirical model in the energy-altitude-latitude system based on studies by Mendall and Korff [81], Armstrong [82], and Merker et al. [83]. A model by Wilson-Nealy [6], based on the other system, is more recent and more comprehensive, but it is not as easy to use as the older model. Taber and Normand believe that the older energy-altitude-latitude model is sufficiently accurate for microelectronics applications.

Since the discovery of SEUs on at aircraft altitudes, researchers have made significant efforts to monitor the environment. Dyer et al. flew a version of their Cosmic 
Ray Environment and Activation Monitor (CREAM) on regular flights on board Concorde G-BOAB between November 1988 and December 1992. At NSREC 1989 Dyer et al. first reported on the results of measurements aboard the Concorde aircraft [84]. Results from 512 flights have been analyzed of which 412 followed high latitude transatlantic routes between London and either New York or Washington DC [71]. Thus some 1,000 hours of observations have been made at altitudes in excess of 50,000 feet and at low cut-off rigidity $(<2$ $\mathrm{GV}^{* *}$ ), and these span a significant portion of solar cycle 22. Fig. 18 shows the count rate in CREAM channel 1 (19fC to $46 \mathrm{fC}$, LET $6.1 \mathrm{MeV} \mathrm{cm}^{2} \mathrm{~g}^{-1}$ ) plotted as monthly averages for the ranges $54,000-55,000$ feet and 1-2 GV. The rates show a clear anticorrelation with the solar cycle and track well with the neutron monitor at Climax Colorado (altitude $3.4 \mathrm{~km}$, cut-off rigidity $2.96 \mathrm{GV})$. The enhanced period during September and October 1989 comprised a number of energetic solar particle events observed by ground level, high latitude neutron monitors and the Concorde observations are summarized in Table $5[84,85]$. The enhancement factors are the ratios between flight- averaged count rates at greater than 50,000 feet during the solar particle events and the rates during immediately preceding flights on identical routes when only quiet-time cosmic rays were present. The charge-deposition thresholds of each of the channels are also given. Recently Dyer and Lei [80] have attempted to calculate the neutron fluxes in the atmosphere during large solar particle events using Monte-Carlo radiation transport in conjunction with data from ground-level neutron monitors and space borne detectors. Some success has been obtained in fitting the results from Concorde and it has been demonstrated that such events can produce high rates of SEU, up to several hundred times the cosmic ray rates, for high latitude routes even at subsonic altitudes of less than 40,000 feet.

TABLE 5: ENHANCEMENT FACTORS FOR CREAM ON CONCORDE DURING SOLAR PARTICLE EVENTS

The CREAM detector has also been operated on a Scandinavian Airlines Boeing 767 operating between Copenhagen and Seattle via Greenland, a route for which the cut-off rigidity is predominately less than 2 GV. Approximately 540 hours of data accumulated between May and August 1993 have been analyzed and these are combined with Concorde data from late 1992 to give updated altitude profiles [3]. Using the AIRPROP code [86], they have also shown that cosmic

\footnotetext{
** Magnetic rigidity of a charged particle is defined as the momentum per unit charge and is measured in units of electron volts/number of charge units, i.e., volts or gigavolts (GV).
}

rays and their secondary fragments are not the major contribution to SEUs at aircraft altitudes. Recent work [87] has concentrated on explaining both the altitude dependence and the energy deposition spectra using a microdosimetry code extension to the Integrated Radiation Transport Suite. Fig. 19 shows that atmospheric secondary neutrons are the major contribution but that ions start to become important at the highest altitudes. Fig. 20 shows that at 30,000 feet the charge deposition spectrum is dominated by neutron interactions at the high end while energetic secondary electrons and muons contribute to the low channels. The work of Normand et al. [88] arrived at similar conclusions on the neutron contribution by scaling results of irradiation of silicon detectors obtained at a spallation neutron source.

\section{B. Ground Environments}

The first evidence of sea-level soft fails (single event upsets) on $16 \mathrm{~Kb}$ DRAMs was given by May and Woods [89]. The source of the radiation was traced to alpha particle emission from contaminates in ceramic packing produced in a factory on the Green River in Colorado which was downstream from an old uranium mine. In 1978 Zeigler realized that, if alpha particles from materials could induce soft fails, it is possible that ground cosmic rays could do the same thing. In 1979 Ziegler and Lanford detailed the mechanism by which sea level cosmic rays could cause soft fails [90]. O'Gorman et al. began field testing of soft fails on memories in the early 1980s. In those experiments, they found a distinct increase in soft fail rates with increasing altitude above sea level [91]. An IBM study on the repair rates of memory modules confirmed the altitude effect on soft failure rate, implying that cosmic radiation was responsible for some of the soft fails on devices in ground systems. The results of that study showed that the failure rates in the mountain states (mean altitude $3200 \mathrm{ft}$ ) was 5 times higher than in the United States as a whole and that the rates in Denver at 5,280 ft. were 10 times higher [92].

There are many products from the cosmic radiation showers (see Section II.B and Fig. 4) so it was important to determine which were dominant in causing the soft fails. At the 1983 NSREC, Dicello concluded that all types of particle radiation could cause soft fails at the ground level with some probability [93]. At NSREC 1996, Normand correlated measured ground level bit error rate with Weapons Neutron Research neutron beam soft fail rate measurements to show that neutrons are the dominant cause of soft fails on the ground [94]. At the 1997 NSREC, Normand et al. showed that neutrons could also cause destructive failures in microelectronics on the ground [95].

Ziegler and colleagues began an effort to define the cosmic ray terrestrial environment based on the work of many researchers who began studying cosmic radiation 
after Hess' balloon experiments [5]. The focus of the work was on defining neutron spectra as a function of altitude as shown in Fig. 21. In the figure the altitude dependence is expressed as atmospheric depth where 0 $\mathrm{g} / \mathrm{cm}^{2}$ represents the top of the atmosphere. Ziegler concludes that the energy spectra of neutrons at various locations through the world are known within a factor of 2 below energies of $100 \mathrm{MeV}$. Two problem areas that he notes are that 1) the accuracy of the levels of neutrons above $100 \mathrm{MeV}$ is no better than a factor of 3 and 2) the flux of neutrons in the energy range of 20$100 \mathrm{MeV}$ is very dependent on local surroundings reducing the accuracy to a factor of 3 .

\section{SUMMARY}

The state of the knowledge of the space, atmospheric, and ground level radiation environments was reviewed, tying it with the last 40 years of the NSREC and its proceedings. We saw that basic science research often is used to derive the definitions required for understanding radiation effects and for developing models that are useful for designing radiation hardened systems. Often our ability to increase system capability is limited by appropriate models of the radiation environment. Large uncertainty factors in environment definition translate to large design margins. The direct result is reduced system resources due to increased shielding, higher mitigation overhead, and/or the use of less capable components.

The availability of useful models of the radiation environment is dependent on our knowledge of the environment, the availability of appropriate data for modeling, and resources for modeling and validation. The use of radiation sensitive modern microelectronics in systems requires models that can meet the needs of system designers and operators through all phases of the life cycle. Due increasing sensitivity of microelectronics to radiation and increasing complexity of spacecraft systems, it is more difficult to completely avoid the risk of radiation effects on systems. The approach is to reduce risk during mission planning and spacecraft and instrument design and to manage the residual risk during launch and operations.

Fig. 22 shows three mission phases that require environment models to address risk issues in systems. Statistically based specification or "climate" models are used for the design phase, which includes system design and mission planning. The minimum requirements of the models are that they should represent long term variation over the solar cycle with at least 1-month resolution, provide worst case estimates, provide confidence levels, and represent the environment in a broad energy range so they are applicable for surface materials to deeply embedded sensors. The operation phase requires "forecasting" models for protecting the systems investment, for mission planning, and for personnel scheduling. The minimum requirements of these models are spatial coverage from interplanetary to low Earth orbits, information about the level of severity of storms, forecasts of quiet times for maneuvers and on-board operations, and they must be specific to effects on technologies. There is increasing awareness of the need to develop forecasting (space weather) models as part of the space infrastructure [9]. The third type of model for "nowcasting" the environment is used to resolve anomalies so risk can be reassessed for both the operating systems and for other systems that are in development. The minimum requirements of these models are spatial coverage from interplanetary to low earth orbit, time profile of the radiation event, adequate energy range and ion composition/linear energy transfer (LET) range. They should also be specific to the effect on technology. If anomaly resolution is critical to the mission and must be performed in near real-time, monitors that are in close proximity to the system may be required.

There are still radiation environments that need improved models. Funding by NASA's Space Environments and Effects Program and the Living with a Star Program and ESA/ESTEC has resulted in improvements in "climate" models of the environment in some areas. However, model consolidation, validation, and standardization has not been supported.

The Living with a Star Program is making heavy investments in developing the scientific understanding of the how the sun-earth system is connected. NASA is also working with organizations that need to nowcast and forecast environments for operational systems to transition that knowledge to operational models. It is hoped that this effort will be extended to include the "climate" models that are required for system design.

\section{ACKNOWLEDGEMENT}

The authors wish to thank Dr. Allan Johnston of the Jet Propulsion Laboratory, the General Chairman of NSREC 2003, for urging the NSREC community to revisit some of the outstanding research that has been published over the past forty years. We also acknowledge the support of Chikia Barnes of SGT Inc. and Martha O'Bryan of Raytheon in producing graphs from archival materials.

\section{VIIII. REFERENCES}

[1] E. G. Stassinopoulos, 1990 IEEE NSREC Short Course, "Microelectronics for the natural radiation environments of space, Chapter I: Radiation Environments of Space," July 16, 1990, Reno, Nevada

[2] J. L. Barth, 1997 IEEE NSREC Short Course, "Applying computer simulation tools to radiation effects problems, Section II: Modeling space radiation environments," 21 July 1997, Snowmass Village, Colorado.

[3] C. S. Dyer, 1998 IEEE NSREC Short Course, "Radiation effects in the new millennium - Old realities and new issues, Section II: Space radiation environment dosimetry." July 20 , 1998, Newport Beach, Califormia. 
[4] J. Mazur 2002 IEEE NSREC Short Course, "Radiation effects - from particles to payloads, Section II: The radiation environment outside and inside a spacecraft," July 15, 2002, Phoenix, Arizona.

[5] J. F. Ziegler, "Terrestrial cosmic rays," IBM Journal of Research and Development, vol. 40, no. 1, pp. 19-39, January 1996.

[6] A. H. Taber and E. Normand, "Investigation and characterization of SEU effects and hardening strategies in avionics," DNA-TR-94-123, Defense Nuclear Agency, Alexandria, VA, February 1995.

[7] M. L. Duldig, "Cosmic ray modulation research," Publications of the Astronomical Society of Australia, vol. 18 , no. 1, pp. 12-40, 2001

[8] N. Drakos, http://cbl.leeds.ac.uk/nikos/tex $2 \mathrm{html} / \mathrm{doc} /$ latex $2 \mathrm{html} / \mathrm{late} 2 \mathrm{ht}$ $\mathrm{ml} . \mathrm{html}$, Computer Based Learning Unit, University of Leeds, 1993.

[9] I. A. Daglis, Space Storms and Space Weather Hazards, vol. 38, Chapter 3, Kluwer Academic Publishers, 2001.

[10] J. A. Van Allen, C. E> Mcllwain, and G. H. Ludwig,

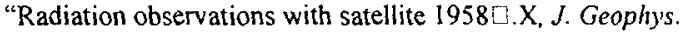
Res. Vol. 64, pp. 271-286, 1959a.

[11] J. I. Vette, "The NASA/National Space Science Data Center trapped radiation environment model program (1964-1991)," NSSDC 91-29, NASA/Goddard Space Flight Center, National Space Science Data Center, Greenbelt, MD, November 1991.

[12] M. J. Teague and E. G. Stassinopoulos, "A model of the starfish flux in the inner radiation zone," X-601-72-487, NASA/Goddard Space Flight Center, Greenbelt, MD, December 1972.

[13] J. I. Vette, "The space radiation environment," IEEE Trans. on Nucl. Sci., vol. 12, no. 5, pp. 1-17, October 1965.

[14] C. E. Mcllwain, "The redistribution of trapped protons during a magnetic storm," Preprint, University of California at San Diego.

[15] D. M. Sawyer and J. I. Vette, "AP-8 trapped proton environment for solar maximum and solar minimum," NSSDC/WDC-A-R\&S, 76-06, NASA/Goddard Space Flight Center, Greenbelt, MD, December 1976.

[16] J. I. Vette, "The AE-8 trapped electron model environment," NSSDC/WDC-A-R\&S 91-24, NASA/Goddard Space Flight Center, Greenbelt, MD, November 1991.

[17] D. S. Beall, C. O. Bostrom, and D. J. Williams, "Structure and decay of the STARFISH radiation belt," October 1963 to December 1965, J. Geophys. Res., vol. 72, pp. 3403-3424, 1967.

[18] C. O. Bostrom, D. S. Beall, and J. C. Armstrong, "Time history of the inner radiation zone," October 1963 to December 1968, J. Geophys. Res., vol. 75, pp. 1246-1256, 1970.

[19] S. L. Brown, "Observations of the artificial radiation belts," in Radiation Trapped in the Earth's Magnetic Field, Astrophysics and Space Science Library, Vol. 5, D. Reidel, Dortrecht, Holland, 1966.

[20] J. A. Van Allen, "Spatial Distribution and time decay of the intensities of geomagnetically trapped electrons from the high altitude nuclear burst of July 1962," in Radiation Trapped in the Earth's Magnetic Field, Astrophysics and Space Science Library, Vol. 5, D. Reidel, Dortrecht, Holland, 1966.

[21] E. G. Stassinopoulos and P. Verzariu, "General formula for decay of Starfish electrons," J. Geophys. Res., vol. 76, pp. 184!-1844, 1971.

[22] E. G. Mullen, M. S. Gussenhoven, K. Ray, and M. Violet, "A double-peaked inner radiation belt: cause and effect as seen on CRRES," IEEE Trans. on Nucl. Sci., vol. 38, no. 6, pp. 1713-1717, December 1991.

[23] M. S. Gussenhoven, E. G. Mullen, and D. H. Brautigam, "Improved understanding of the earth's radiation belts from the CRRES satellite," IEEE Trans. on Nucl. Sci., vol. 43, no 2, pp. 353-368, April 1996.
[24] X. Li, I. Roth, M. Tomerin, J. R. Wygant, M. K. Hudson, and J. B. Blake, "Simulation of the prompt energization and transport of radiation belt particles during the March 24, 1991 SSC," Geophys. Res. Lett., Vol. 20, p. 2423-2426, 1993.

[25] M. K. Hudson, A. D. Kotelnikov, X. Li, I. Roth, M. Temerin, J. Wygant, J. B. Blake, and M. S. Gussenhoven, "Simulation of proton radiation belt formation during the March 24, 1991 SSC," Geophys. Res. Lett., Vol. 22, No. 3, pp. 291-294, February 1, 1995.

[26] D. Boucher, S. Bourdaire, and T. Beutier, "Dynamic modeling of trapped particles," IEEE Trans. on Nucl. Sci., vol. 43, no. 2, pp. 416-425, April 1996.

[27] D. Boscher and S. Bourdarie, "Modeling the radiation belts: what are the important physical processes to be taken into account in models?," Adv. Space Research, 28-12, 1739, 2001.

[28] Private Communication with S. Bourdarie, ONERA, Toulouse, France, September 2002.

[29] E. J. Daly, J. Lemaire, D. Heynderickx, and D. J. Rodgers, "Problems with models of the radiation belts," IEEE Trans. on Nucl. Sci., vol. 43, no. 2, pp. 403-415, April 1996.

[30] M. S. Gussenhoven, E. G. Mullen, M. D. Violet, C. Hein, J Bass, and D. Madden, "CRRES high energy proton flux maps," IEEE Trans. on Nucl. Sci., vol. 40, no. 6, pp. 1450 1457, December 1993

[31] D. H. Brautigam, M. S. Gussenhoven, E. G. Mullen, "Quasistatic model of outer zone electrons," IEEE Trans. on Nucl. Sci., vol. 39, no. 6, pp. 1797-1803, December 1992.

[32] M. S. Gussenhoven, E. G. Mullen, M. Sperry, K. J. Kerns, "The effect of the March 1991 storm on accumulated dose for selected orbits: CRRES dose models," IEEE Trans. on Nucl. Sci., vol. 39, no. 6, pp 1765-1772, December 1992.

[33] M. S. Gussenhoven, E. G. Mullen, J. T. Bell, D. Madden, and E. Holeman, "APEXRAD: low altitude orbit dose as a function of inclination, magnetic activity and solar cycle," IEEE Trans. on Nucl. Sci., vol. 44, no. 6, pp. 2161-2168, December 1997.

[34] K. A. Pfitzer, "Radiation dose to man and hardware as a function of atmospheric density in the 28.5 degree Space Station orbit," MDSSC Rep. H5387, McDonnell Douglas Space Systems Co., Huntington Beach, CA, 1990.

[35] D. Heynderickx, (17 April 1997), Belgisch Instituut voor Ruimte-Aëronmie/Institut d'Aéronomie Spatiale de Belgique (BIRA/IASB), Proceedings from Model Workshop, "Low altitude trapped radiation model using TIROSNNOAA data" by S. L. Huston, G. A. Kuck, and K. A. Pfitzer, [WWW document], URL http://magnet.oma.be/trend/trend2.html

[36] S. L. Huston and K. A. Pfitzer, "A new model for the low altitude trapped proton environment," IEEE Trans. on Nucl. Sci., vol. 45, no. 6, pp. 2972-2978, December 1998.

[37] Mi. A. Xapsos, S. L. Huston, J. L. Barth, and E. G. Stassinipoulos, "Probabilistic model for low-altitude trapped proton fluxes," IEEE Tians. on Nucl. Sci, vol. 49, no. 6, pp 2776-2781, December 2002.

[38] D. Heynderickx and J. Lamaire, "Coordinate systems for mapping low-altitude trapped particle fluxes," Proc. Taos Workshop on Earth's Trapped Particle Environments, Aug. 14-19, 1994.

[39] D. Heynderickx, M. Kruglanski, V. Pierrard, J. Lemaire, M. D. Looper, and J. B. Blake, "A low altitude trapped proton model for solar minimum conditions based on SAMPEX/PET data," IEEE Trans. on Nucl. Sci., vol. 46, no. 6, pp. 14751480, December 1999.

[40] J. W. Haffner, "Natural nuclear radiation environments for the Grand Tour missions," IEEE Trans. on Nucl. Sci., vol. 18, no. 6, pp. 443-453, December 1971

[41] P. G. Kase, "The radiation environments of outer-planet missions," IEEE Trans. on Nucl. Sci., vol. 19, no. 6, pp. 141146, December 1972.

[42] N. Divine, "The Planet Jupiter (1970)," NASA SP-8069, Jet Propulsion Laboratory, Pasadena, California, December 1971. 
[43] From the History of NASA, SP-349/396 Pioneer Odyssey, http://history.nasa.gov/SP-349/ch5.htm.

[44] Private communication with $T$. Oldham on talk by $G$. Lee at the 1983 Single Event Effects Symspoium, QSS Group at Goddard Space Flight Center, Code 561, Greenbelt, MD 20771, January 2003.

[45] N. Divine and H. B. Garrett, "Charged particle distributions in Jupiter's magnetosphere," J. of Geo. Phys. Res., vol. 88, no. A9, pp 6889-6903, September 1983.

[46] H. H. Malitson and W. R. Webber, "A summary of solar cosmic ray events," Solar Proton Manual, NASA TR R-169.

[47] J. B. Weddel and J. W. Haffner, "Statistical evaluation of proton radiation from solar flares", Report SID 66-421, North American Aviation, July 1966

[48] J. H. King, "Solar proton fluences for 1977-1983 space missions," J. Spacecraft and Rockets, vol. 11 , no. 6, pp. 401408, June 1974.

[49] E. G. Stassinopoulos, "SOLPRO: A Computer Code to Calculate Probabilistic Energetic Solar Flare Protons," NSSDC 74-11, NASA/Goddard Space Flight Data Center, Greenbelt, MD, April 1975.

[50] J. Feynman, G. Spitale, J. Wang, and S. Gabriel, "Interplanetary fluence model: JPL 1991," J. Geophys. Res., vol. 98, pp 13281-13294, 1993.

[51] M. A. Xapsos, J. L. Barth, E. G. Stassinopoulos, G. P. Summers, E. A. Burke, G. B. Gee, "Model for prediction of solar proton events," Proceedings from the Space Radiation Environment Workshop, Farnborough, UK, 1-3 November 1999.

[52] M. A. Xapsos, G. P. Summers, P. Shapiro, and E. A. Burke, "New techniques for prediction solar proton fluences for radiation effects applications," IEEE Trans. on Nucl. Sci., vol. 43, no. 6, December 1996.

[53] M. A. Xapsos, G. P. Summers, and E. A. Burke, "Probability model for peak fluxes of solar proton events," IEEE Trans. on Nucl. Sci., vol. 45, no. 6, pp. 2948-2953, December 1998.

[54] M. A. Xapsos, G. P. Summers, J. L. Barth, E. G. Stassinopoulos, and E. A. Burke, "Probability model for worst case solar proton event fluences," IEEE Trans. on NuCl. Sci., vol. 45, no. 6, pp. 1481-1485, December 1999.

[55] A. J. Tylka, F. Dietrich, and P. R. Boberg, "CREME96: A revision of the cosmic ray effects on microelectronics code," IEEE Trans. on Nucl. Sci., vol. 44, no. 6, pp. 2150-2160, December 1997.

[56] M. A. Xapsos, J. L. Barth, E. G. Stassinopoulos, E. A. Burke, and G. B. Gee, "Model for emission of solar protons (ESP) cumulative and worst case event fluences," NASA-Marshall Space Flight Center SEE Program, http://see.msfc.nasa.gov.

[57] R. A. Millikan, Presentation before the National Academy of Sciences, November 9, 1925, Madison, Wisconsin.

[58] P. J. McNulty, "Single event effects experienced by astronauts and microelectronic circuits flown in space," IEEE Trans. on Nucl. Sci., vol. 43, no. 2, pp. 475-482, April 1996.

[59] D. Binder, E. C. Smith, and A. B. Holman, "Satellite anomalies from galactic cosmic rays," IEEE Trans on Nucl. Sci., vol. 22, no. 6, pp. 2675-2680, December 1975.

[60] P. Meyer. R. Ramaty, and W. R. Weber, Physics Today, vol. 27, no. 10, 231974.

[61] M. O. Burrell and J. J. Wright, "The estimation of galactic cosmic ray penetration and does rates," NASA TN D-6600, March 1972.

[62] J. H. Adams, Jr., R. Silberberg, and C. H. Tsao, "Cosmic ray effects of microelectronics, Part I: The near-earth particle environment," NRL Memorandum Report 4506, August 25. 1981

[63] J. H. Adams, Jr., J. R. Letaw, and D. F. Smart, "Cosmic ray effects of microelectronics, Part II: Geomagnetic cutoff effects," NRL Memorandum Report 5099, May 26, 1983.
[64] J. H. Adams, Jr., "The natural radiation environment inside spacecraft," IEEE Trans. on Nucl. Sci., Vol. 29, No. 6, pp. 2095-2100, December 1982.

[65] E. L. Peterson, P. Shapiro, J. H. Adams, Jr., and E. A. Burke, "Calculation of cosmic-Ray Induce Soft Upsets and Scaling in VLSI Devices," IEEE Trans. on Nucl. Sci., Vol. 29, No. 6, pp. 2055-2063, December 1982.

[66] W. Heinrich, Radiation Effects, vol. 34, no. 143, 1977.

[67] E. L. Peterson, "Approaches to proton single-event rate calculations," IEEE Trans on Nucl. Sci., vol. 43, no. 2, pp. 496-504, April 1996.

[68] J. C. Pickel and J. T. Blanford Jr., "Cosmic ray induced errors in MOS memory cells," IEEE Trans. on Nucl. Sci., vol. 25, no. 6, pp. 1166, December 1978

[69] J. H. Adams, Jr., "Cosmic ray effects on microelectronics, Part IV," NRL Memorandum Report 5901, Naval Research Laboratory, Washington, DC, December 31, 1986.

[70] D. V. Reames, H. V. Cane, and $T$. T. von Rosenvinge, "Energetic particle abundances on solar electron events," The Astrophys. J., vol. 357, pp. 259-270, July I, 1990.

[71] A. Sims, C. Dyer, C. Peerless, K. Johansson, H. Pettersson, and J. Farren, "The single event upset environment for avionics at high latitude," IEEE Trans. on Nuc. Sci., vol. 41, no. 6, pp. 2361-2367, December 1994.

[72] A. J. Tylka, F. Dietrich, and P. R. Boberg, "Probability distributions of high-energy solar-heavy-ion fluxes from IMP8: 1973-1996," IEEE Trans. on Nucl. Sci., vol. 44, no. 6, pp. 2140-2149, December 1997.

[73] C. S. Dyer, C. Sanderson, R. Mugford, C. Watson, C. Peerless, "Radiation environment of the Microelectronics and Photonics Test Bed as measured by CREDO-3," IEEE Trans. on Nucl. Sci., vol. 47, no.3, pp. 481-485, June 2000.

[74] C. S. Dyer, K. Hunter, S. Clucas, D. Rodgers, A. Campbell, S. Buchner, "Observation of solar particle events from CREDO and MPTB during the current solar maximum," IEEE Trans. on Nucl. Sci., vol. 49, no. 6, pp. 2771-2775, December 2002.

[75] C. H. Tsao, R. Silberberg, and J. R. Letaw, "Cosmic ray heavy ions at and above 40,000 feet," IEEE Trans. on Nucl. Sci., vol. 31, no. 6, pp. 1066-1068, December 1984.

[76] C. H. Tsao, R. Silberberg, H. H. Adams Jr., and J. R. Letaw, "Cosmic ray effects on microelectronics, part 3: Propagation of cosmic rays in the atmosphere", NRL Memorandum Report 5402, August 1984.

[77] R. Silberberg, C. H. Tsao, J. R. Letaw, "Neutron generated single event upsets," IEEE Trans. on Nucl. Sci., vol. 31, No 6, pp. 1183-1185, December 1984.

[78] J. Olsen, P. E. Becher, P. B. Fynbo, P. Raaby, and J. Schultz, "Neutron induced single event upset on static RAMs observed at $10 \mathrm{~km}$ flight altitude," IEEE Trans. on Nucl. Sci., vol. 40, no. 2, pp. 74-77, April 1993 .

[79] A. Taber and E. Normand, "Single event upset in avionics," IEEE Trans. on Nucl. Sci., vol. 40, pp. 120, April 1993.

[80] C. S. Dyer and F. Lei, "Monte-Carlo calculations of the influence on aircraft radiation environments of structures and solar particle events," IEEE Trans. on Nucl. Sci.,vol. 48, no. 6, pp. 1987-1995, Dec. 2001.

[81] R. B. Mendall and S. A. Korff, "Fast neutron flux in the atmosphere," J. Geophys., vol. 68, 5487, 1963.

[82] T. W. Armstrong et al., "Calculation of neutron flux spectra induced in the earth's atmosphere by galactic cosmic rays," $J$. Geophys. Res., vol. 78, 2715, 1973.

[83] M. Merker et al., "Time dependent world-wide distribution of atmospheric neutrons and their products," J. Geophys. Res., vol. 78, 2727, 1973.

[84] C. S. Dyer, A. J. Sims, J. Farren, and J. Stephen, "Measurements of the SEU environment in the upper atmosphere," IEEE Trans. on Nuc. Sci., vol. 36, no. 6, pp. 2275-2280, December 1989.

[85] C. S. Dyer, A. J. Sims, J. Farren, J. Stephen, and C. Underwood, "Comparative measurements of the single event upset and total dose environments using the CREAM 
instruments," IEEE Trans. on Nuc. Sci., vol. 39, no. 3, pp. 413-417, June 1992.

[86] C. Tsao, R. Silberberg, J. Adams Jr., and J. Letaw, "Cosmic ray effects on microelectronics: Part III: Propagation of cosmic rays in the atmosphere," NRL Memorandum Report 5402, Aug 1984.

[87] C. S. Dyer and P. R. Truscott, "Cosmic radiation effects on avionics," ERA Technology Conference Volume for 1997 Avionics Conference, Heathrow UK, pp 6.3.1-6.3.10, November 1997.

[88] E. Normand, D. Olberg, J. Wert, J. Ness, P. Majewski, S. Wendron, and A. Gavron, "Singie event upset and charge collection measurements using high energy protons and meutrons," IEEE Trans. on Nul. Sci., vol. 41 , no. 6, pp. 2203 2209, Dec 1994

[89] T. C. May and M. H. Woods, "Alpha-particle-induced soft errors on dynamic memories," IEEE Trans. Electron Devices, vol. 26, pp. 2-9, January 1979.

[90] J. F. Ziegler and W. A. Lanford, SCIENCE, vol. 206, pp. 776, 1979.

[91] T. J. O'Gorman, J. M. Ross, A. H. Taber, J. F. Ziegler, H. P. Muhlfeld, C. J. Montrose, H. W. Curtis, and J. L. Walsh, "Field testing for cosmic ray soft errors in semiconductor memories," IBM Journal of Research and Development, vol. 40, no. 1, pp. 41-50, January 1996.

[92] J. F. Ziegler, H. W. Curtis, H. P. Muhlfeld, C. J. Montrose, B. Chin, M. Nicewicz, C. A. Russell, W. Y. Wang, L. B. Freman, P. Hoiser, L. E. LaFave, J. L. Walsh, J. M. Orro, G. J. Unger, J. M. Ross, T. J. O'Gorman, B. Messine, T. D. Sullivan, A. J. Sykes, H. Yourke, T. A. Enger, V. Toalt, T. S. Scott, A. H. Taber, R. J. Sussman, W. A. Klein, and C. W. Wahaus, "JBM experiments in soft fails in computer electronic (1978-1994)," IBM Journal of Research and Development, vol. 40, no. 1, pp. 3-18, January 1996.

[93] J. F. Dicello, C. W. McCabe, J. D. Doss, and M. Paciotti, "The relative efficiency of sofi-error induction in $4 \mathrm{~K}$ static RAMs by muons and pions," IEEE Trans. on Nucl. Sci., vol. 30, no. 6, pp. 4613-4615, December 1983.

[94] E. Normand, "Single event upset at ground level," IEEE Trans. on Nucl. Sci., vol. 43, no. 6, pp. 2742-2750, December 1996.

[95] E. Normand, J. L. Wert, D. L. Oberg, P. P. Majewski, P. Voss, and S. A. Wender, "Neutron-induced single event burnout in high voltage electronics," IEEE Trans. on Nucl. Sci., vol. 44, no. 6, pp. 2358-2366, December 1997.

Fig. 1: Artist's drawing of the Earth's Van Allen radiation belts. The drawing does not show the SAA. from BIRA

Fig. 2: Radiation belt electrons ( $\mathrm{E}>0.4 \mathrm{MeV}$ ) measured by the SAMPEX spacecraft. The measurements are plotted as a function of $L$ and the day of year. The outer zone $(L>3)$, slot region ( $L=2-3)$, and the inner zone $(L<3)$ can be clearly seen. The plot shows the dynamic electron population in the outer zone and the numerous episodes of the slot region being filled with storm electrons. S. Kanekal, NASA/GSFC

Fig. 3: The IMP-8 measurements of carbon, oxygen, and nitrogen ions in interplanetary space (24-hour averaged mean exposure flux). Also plotted is the average sunspot counts for the same time period. Note the sudden rises in the counts due to solar particle events and the anticorrelation of the galactic cosmic ray levels with the sunspot counts.

Fig. 4: Cosmic rays hit the top of the atmosphere and disintegrate into neutrons, pions, muons, etc. Smart and Shea

Fig. 5: Components in the ground level cosmic ray environment at New York City as a function of energy, Zeigler $/ I B M$
Fig. 6: The AP-3 proton model map presented by Vette at NSREC 1965

Fig. 7: Dependence by region of the decay lifetime for electrons in the STARFISH model

Fig. 8: Decay cutoff times in months for the STARFISH model as a function of threshold energy and $L$

Fig. 9: Integral Van Allen belt electrons before and after the Soviet event

Fig. 10: Schematic of the distribution of fission electrons from the STARFISH and Soviet tests in magnetic coordinates

Fig. 11: Comparison of the average lifetimes of $>2 \mathrm{MeV}$ electrons for the STARFISH and Soviet tests

Fig. 12: The second proton belt produced by the March 1991 magnetic storm measured by CRRES for proton energies of $47 \mathrm{MeV}$, AFRL/VS

Fig. 13: Results from the Salammbo 4-D diffusion code showing the distribution of $100 \mathrm{keV}$ electrons after an injection (storm) event. The omnidirectional, differential fluxes are in units of $\mathrm{MeV}^{-1} \mathrm{~cm}^{-2} \mathrm{~s}^{-1}$. Bourdarie/ONERA

Fig. 14: Steep gradients in the AP-8 model at low L values for integral proton fluxes at $E>30 \mathrm{MeV}$. The fluxes are omnidirectional and integral for $\mathrm{E}>0.5 \mathrm{MeV}$. Daly/ESA-ESTEC

Fig. 15: Proton distributions on the magnetic equator for protons in the inner zone at $E>80 \mathrm{MeV}$ which were used as the basis for the first trapped proton model with true solar cycle dependence. Huston and Pfitzer

Fig. 16: Comparison of probability models for solar particles

Fig. 17: Comparison of CREME86 and CREME96 solar heavy ions models for a geostationary orbit

Fig. 18: Monthly mean count rates from the CREAM instrument on Concorde from Jan 1989 to Dec 1992 compared with ground level neutron monitor at Climax

Fig. 19: Average CREAM channel 5 count rates as a function of altitude at 1-2 GV from SAS and Concorde flights. Also shown are the predictions from AIRPROP and from neutron interactions as calculated using radiation transport and microdosimetry codes (LHI IMDC). Neutrons dominale at 3,000 to 40,000 feet but cosmic ray ions contribute at supersonic altitudes.

Fig. 20: The spectrum of charge deposition from CREAM at 30,00031,000 feet compared with predictions. Neutrons dominate at high values while electrons and muons contribute at the low end

Fig. 21: "Hess values" of measured energy spectra of neutrons at various altitudes up to outer space. The lowest curve at $1030 \mathrm{~g} / \mathrm{cm}^{2}$ is at sea level and the curve marked $700 \mathrm{~g} / \mathrm{cm}^{2}$ is at an altitude of 2 miles. Zeigler

Fig. 22: Environment model types that are required in each phase of risk management for missions 
TABLE 1: MAXIMUM ENERgIES OF PARTICleS

\begin{tabular}{ll}
\hline Particle Type & Maximum Energy \\
\hline Trapped Electrons & 10 s of MeV \\
Trapped Protons \& Heavy Ions & 100 s of MeV \\
Solar Protons & $\mathrm{GeV}$ \\
Solar Heavy Ions & $\mathrm{GeV}$ \\
Galactic Cosmic Rays & $\mathrm{TeV}$ \\
\hline
\end{tabular}


TABLE 2: ANNUAL DOSE FROM GROUND RADIATION IN MSv [8]

\begin{tabular}{ccc}
\hline Source & Type & Annual Dose \\
\hline Terrestrial & Natural & 0.26 \\
Inhaled radionuclides $^{1}$ & Natural & 1.0 \\
Internal radionuclides ${ }^{2}$ & Natural & 0.26 \\
Cosmic radiation & Natural & 0.28 \\
Cosmogenic radionuclides & Natural & 0.01 \\
Medical diagnostics & Manmade & 0.92 \\
Atmospheric weapons testing & Manmade & 0.05 \\
Airline travel & Natural & $1.6 / \mathrm{crew}$ \\
& & $0.03 /$ passenger \\
Consumer products & Manmade & 0.04 \\
Nuclear power & Manmade & $<0.01$ \\
\hline
\end{tabular}

Tust particles bearing radon daughters expose lung tissue to alpha radiation.

${ }^{2}$ Naturally occurring radioactive elements (primarily potassium-40) other than radon daughters that find their way into our bodies 
TABLE 3: ACCURACY OF THE STARFISH DECAY MODEL IN MONTHS

\begin{tabular}{llllcccc}
\hline \multicolumn{7}{c}{ Energy (MeV) } \\
\hline $\mathrm{L}$ & .03 & .05 & .1 & 0.3 & 0.5 & 1.0 & 3.0 \\
1.3 & \pm 4 & \pm 4 & \pm 4 & \pm 4 & \pm 5 & \pm 8 & \pm 10 \\
1.4 & \pm 3 & \pm 3 & \pm 3 & \pm 3 & \pm 4 & \pm 8 & \pm 10 \\
1.5 & \pm 3 & \pm 3 & \pm 3 & \pm 3 & \pm 4 & \pm 8 & \pm 10 \\
1.6 & \pm 3 & \pm 3 & \pm 3 & \pm 3 & \pm 4 & \pm 6 & \pm 7 \\
1.7 & \pm 3 & \pm 3 & \pm 3 & \pm 3 & \pm 4 & \pm 8 & \pm 8 \\
1.8 & \pm 3 & \pm 3 & \pm 3 & \pm 3 & \pm 6 & \pm 8 & \\
1.9 & \pm 8 & \pm 7 & \pm 5 & \pm 5 & \pm 7 & \pm 8 & \\
2.0 & \pm 8 & \pm 7 & \pm 5 & \pm 6 & \pm 8 & \pm 9 & \\
\hline
\end{tabular}


TABLE 4: SOLAR PRotons $>10 \mathrm{MEV}$

\begin{tabular}{ccc}
\hline Year & $\begin{array}{c}\text { Predicted } \\
\mathrm{p} / \mathrm{cm}^{2} / \mathrm{yr}\end{array}$ & $\begin{array}{c}\text { Actual } \\
\mathrm{p} / \mathrm{cm}^{2} / \mathrm{yr}\end{array}$ \\
\hline 1972 & $7.0 \times 10^{9}$ & $2.4 \times 10^{10}$ \\
1989 & $8.0 \times 10^{8}$ & $4.5 \times 10^{10}$ \\
\hline
\end{tabular}


TABLE 5: ENHANCEMENT FACTORS FOR CREAM ON CONCORDE DURING SOLAR PARTICLE EVENTS

\begin{tabular}{cccccc}
\hline $\begin{array}{c}\text { Channel } \\
\text { Number \& } \\
\text { Charge } \\
\text { Deposition } \\
\text { Threshold }\end{array}$ & $\begin{array}{c}29 \text { Sep } \\
1406-1726\end{array}$ & $\begin{array}{c}19 \text { Oct } \\
1420-1735\end{array}$ & $\begin{array}{c}20 \text { Oct } \\
0859-1204\end{array}$ & $\begin{array}{c}22 \text { Oct } \\
1814-2149\end{array}$ & $\begin{array}{c}24 \text { Oct } \\
1805-2135\end{array}$ \\
\hline $119 \mathrm{fC}$ & $3.7 \pm 0.02$ & $1.6 \pm 0.01$ & $1.4 \pm 0.01$ & $1.5 \pm 0.01$ & $3.4 \pm 0.01$ \\
$246 \mathrm{fC}$ & $4.9 \pm 0.1$ & $1.9 \pm 0.04$ & $1.6 \pm 0.04$ & $1.8 \pm 0.04$ & $4.5 \pm 0.06$ \\
$3110 \mathrm{fC}$ & $5.7 \pm 0.1$ & $2.1 \pm 0.07$ & $1.8 \pm 0.07$ & $1.9 \pm 0.07$ & $5.2 \pm 0.1$ \\
$4260 \mathrm{fC}$ & $5.9 \pm 0.2$ & $2.0 \pm 0.1$ & $1.8 \pm 0.1$ & $2.0 \pm 0.1$ & $5.7 \pm 0.2$ \\
$5610 \mathrm{fC}$ & $5.6 \pm 0.6$ & $2.0 \pm 0.3$ & $2.0 \pm 0.4$ & $2.1 \pm 0.3$ & $4.9 \pm 0.4$ \\
$61.50 \mathrm{pC}$ & $6.1 \pm 1.5$ & $3.0 \pm 0.7$ & $1.1 \pm 0.8$ & $1.0 \pm 0.6$ & $4.3 \pm 1.1$ \\
$73.40 \mathrm{pC}$ & $(17.4 \pm 17.4)$ & - & $(30.4 \pm 30.4)$ & - & - \\
$88.10 \mathrm{pC}$ & - & - & - & - & - \\
$919.3 \mathrm{pC}$ & - & - & - & - & - \\
\hline
\end{tabular}



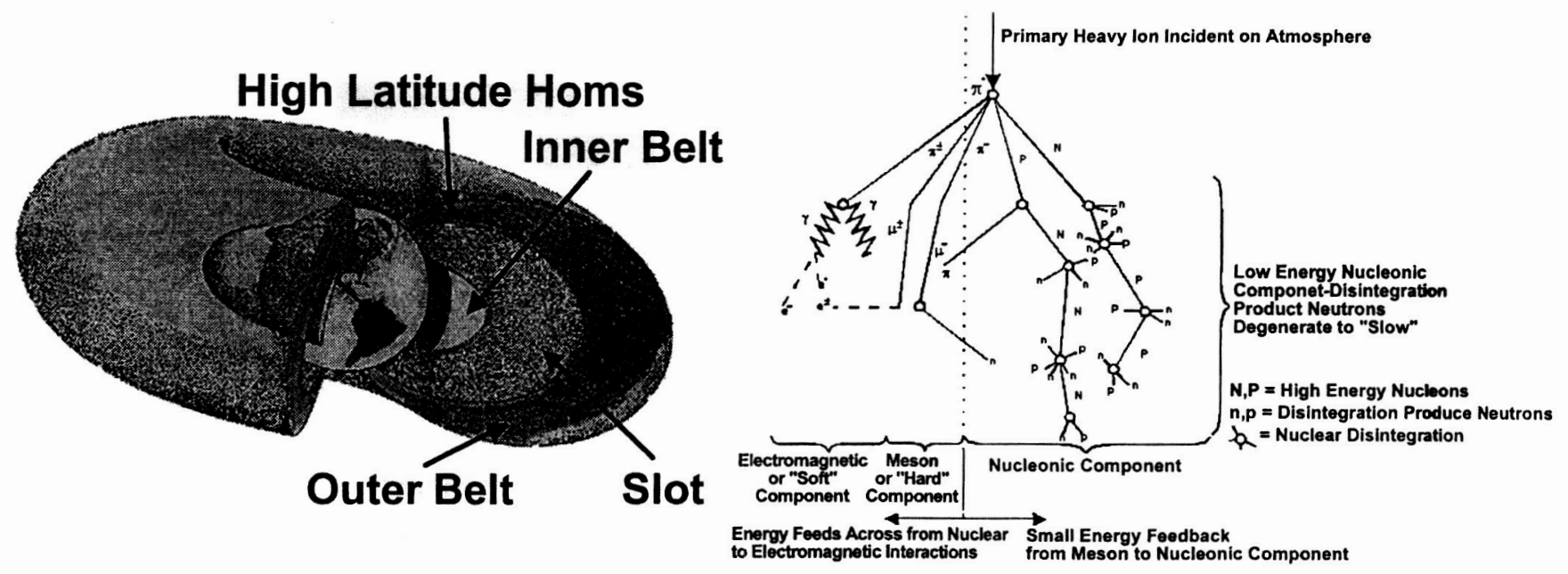

Fig. 1 - See cap. in Paper

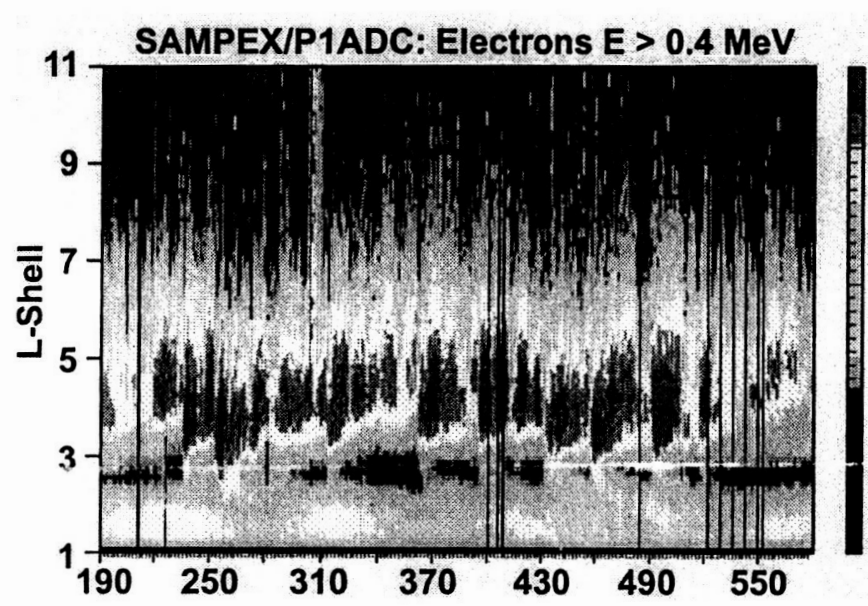

Fig. 4 - See cap. in Paper

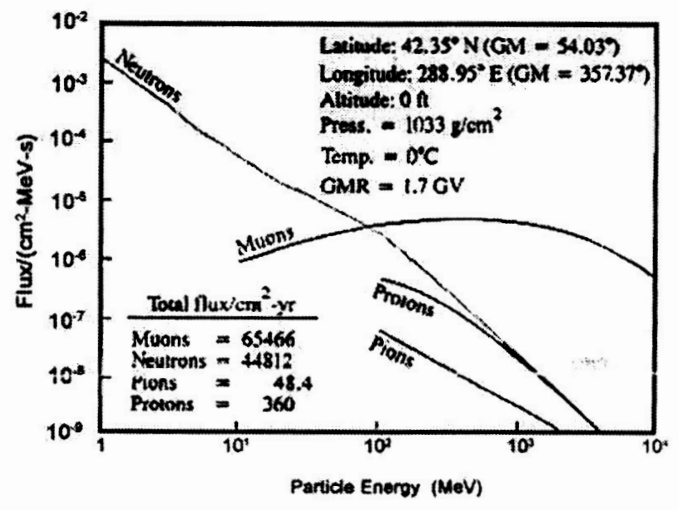

Fig. 2 - See cap. in Paper

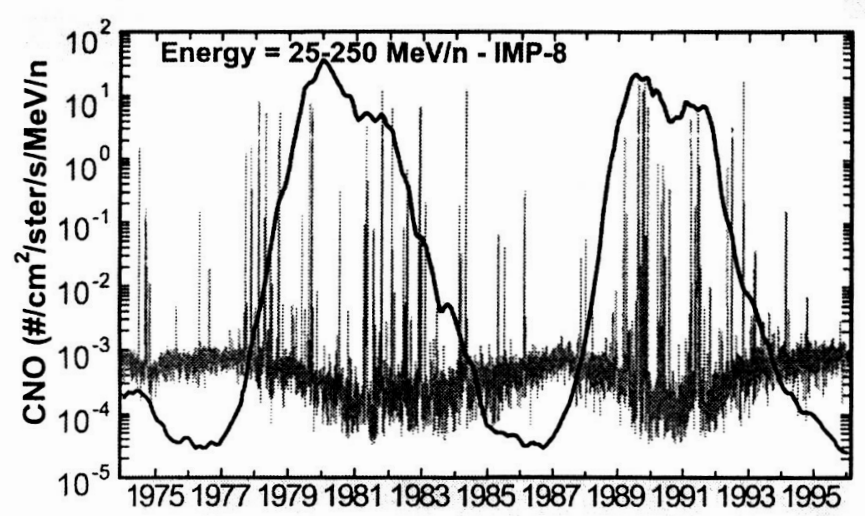

Fig. 5 - See cap. in Paper

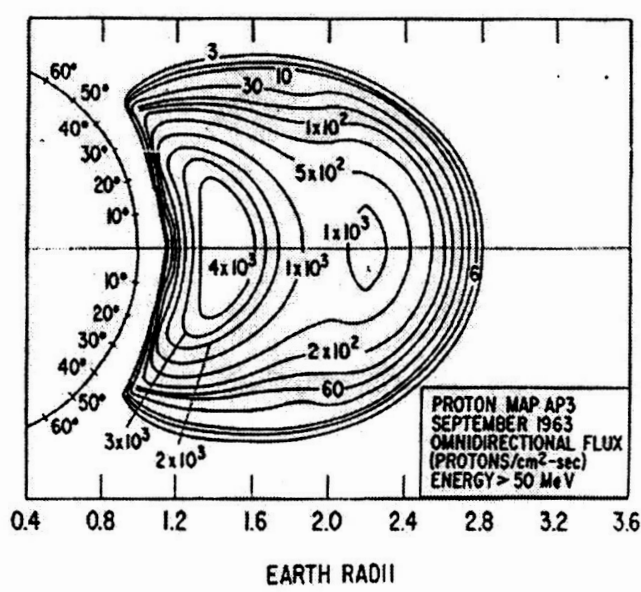

Fig. 3 - See cap. in Paper

Fig. 6 - See cap. in Paper 


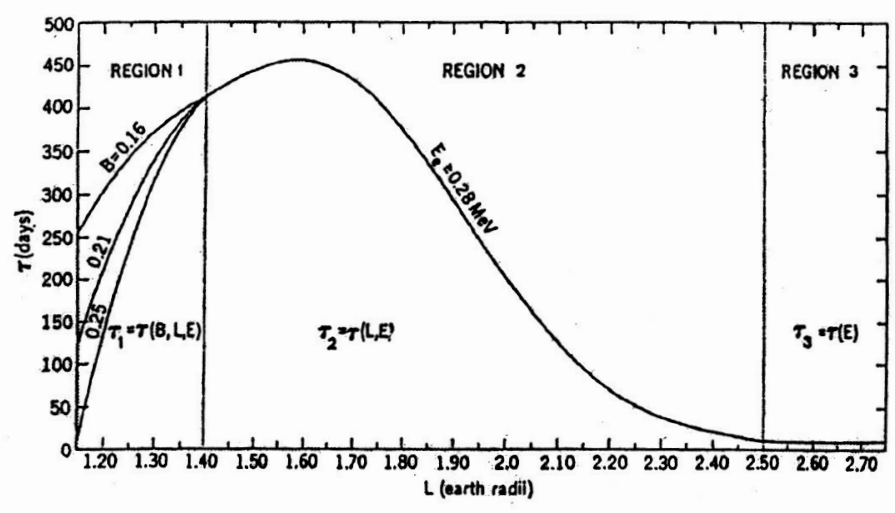

Fig. 7 - See cap. in Paper

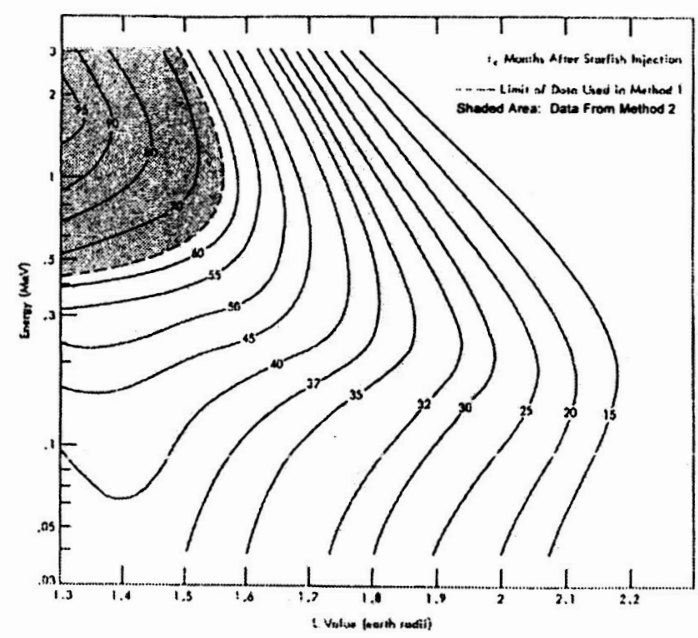

Fig. 8 - See cap. in Paper

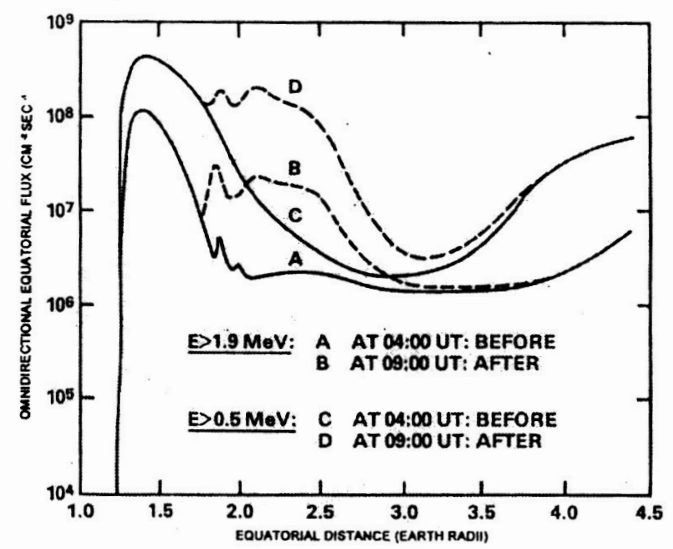

Fig. 9 - See cap. in Paper

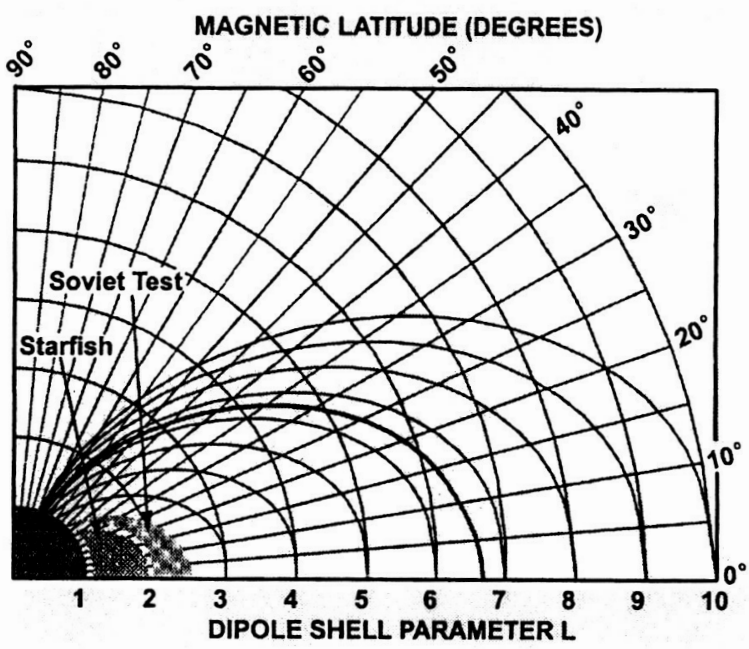

Fig. 10 - See cap. in Paper

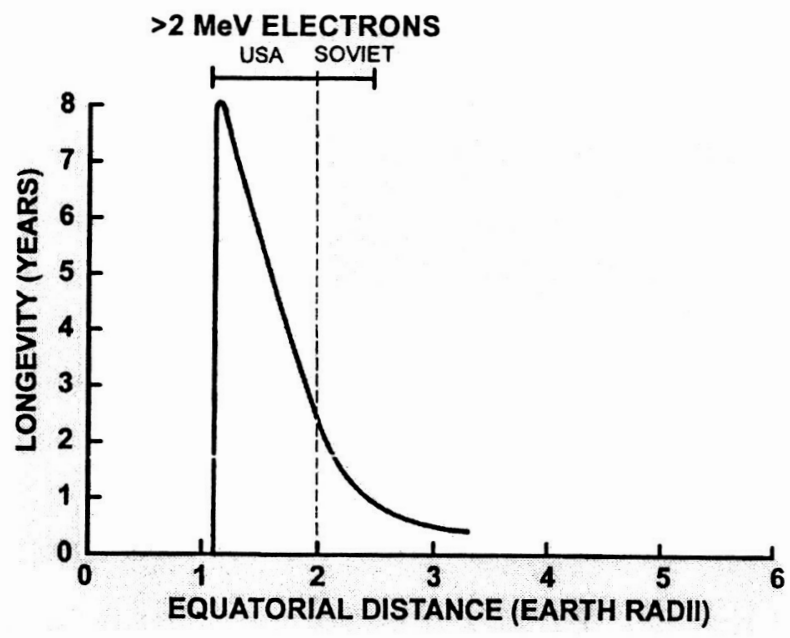

Fig. 11 - See cap. in Paper

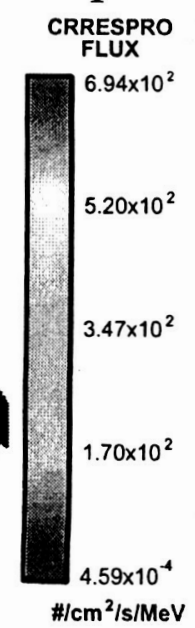

Fig. 12 - See cap. in Paper 
Flux Differential Omindirectional (MeV-1 cm -2 s-1)

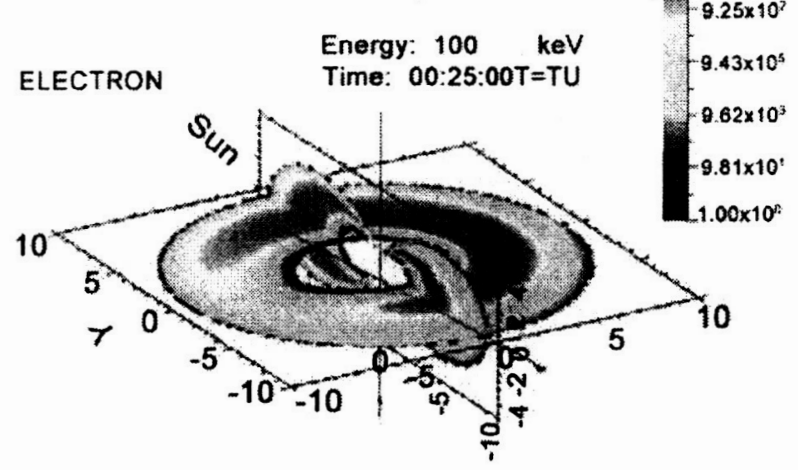

Fig. 13 - See cap. in Paper

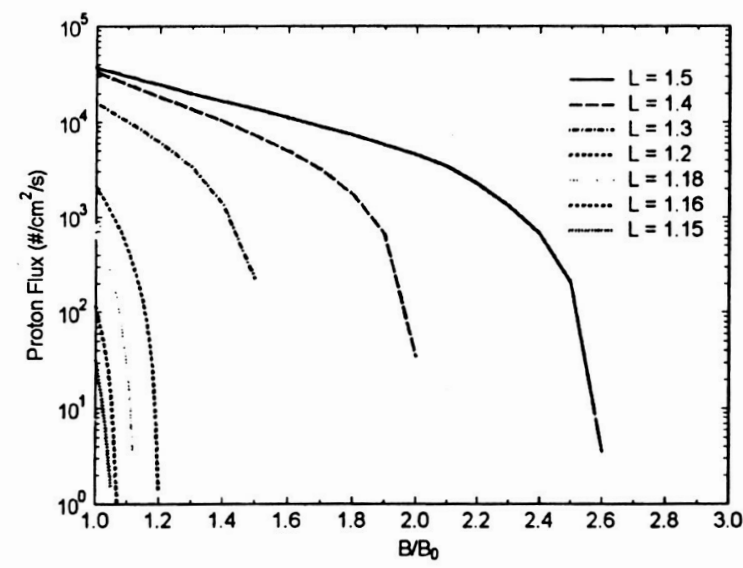

Fig. 14 - See cap. in Paper

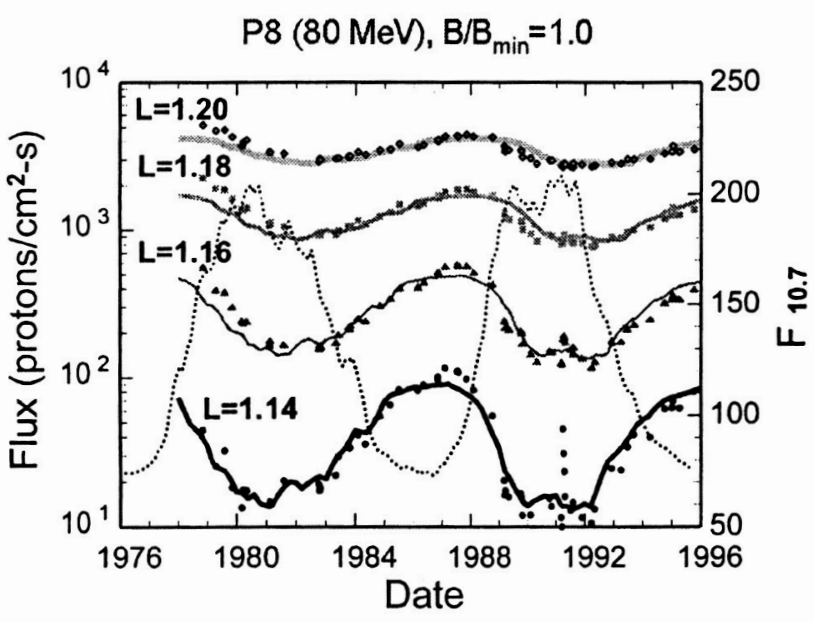

Fig. 15 - See cap. in Paper

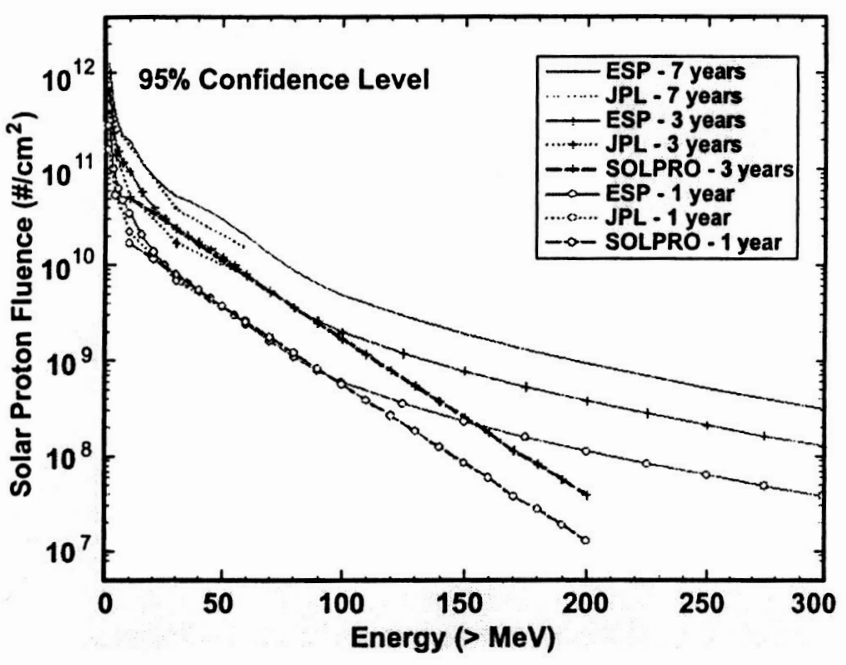

Fig. 16 - See cap. in Paper

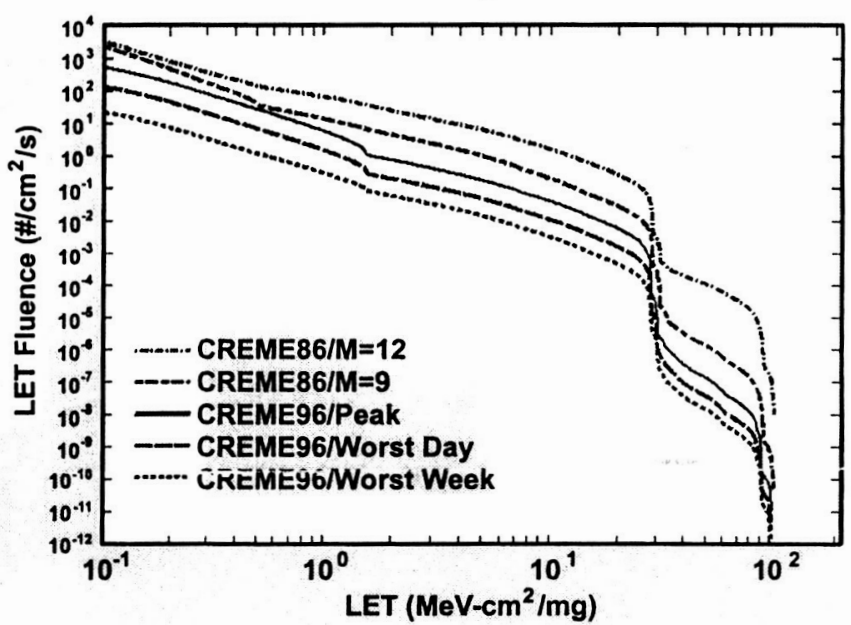

Fig. 17 - See cap. in Paper

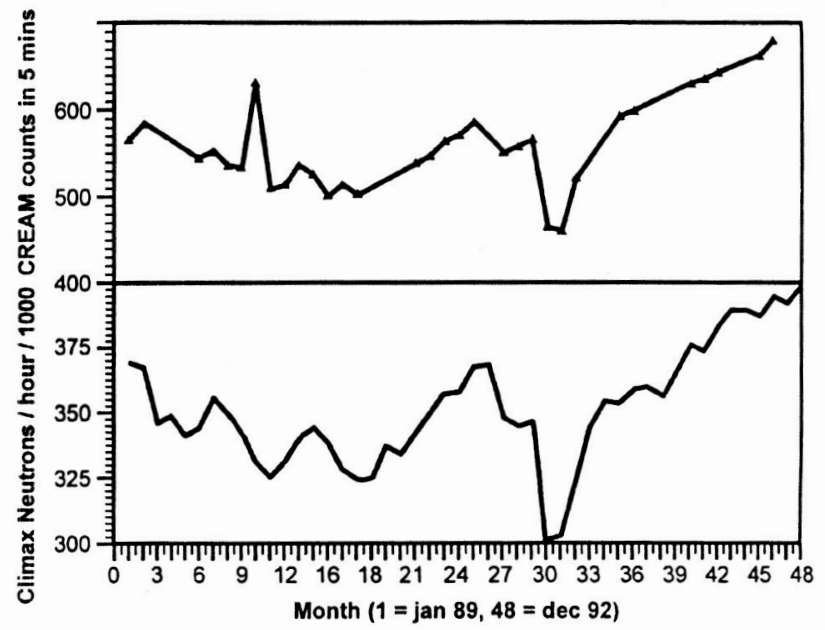

Fig. 18 - See cap. in Paper 


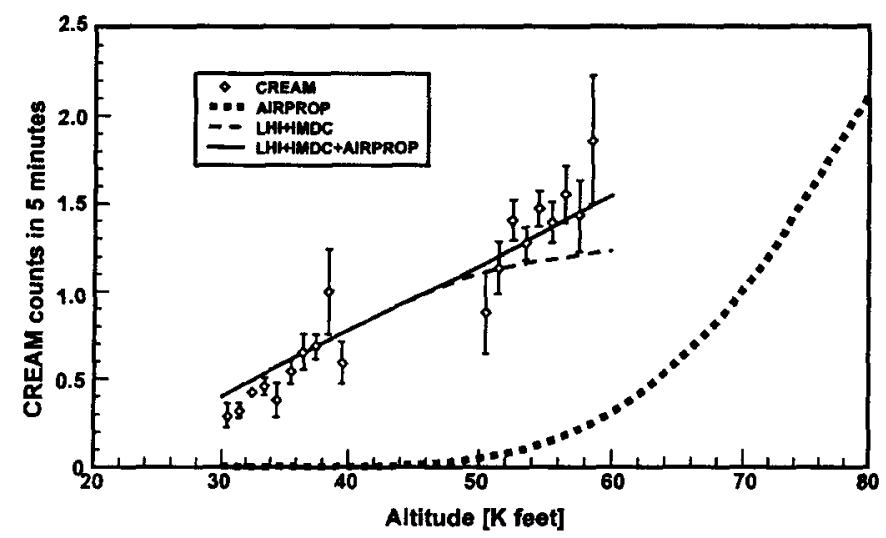

Fig. 19 - See cap. in Paper

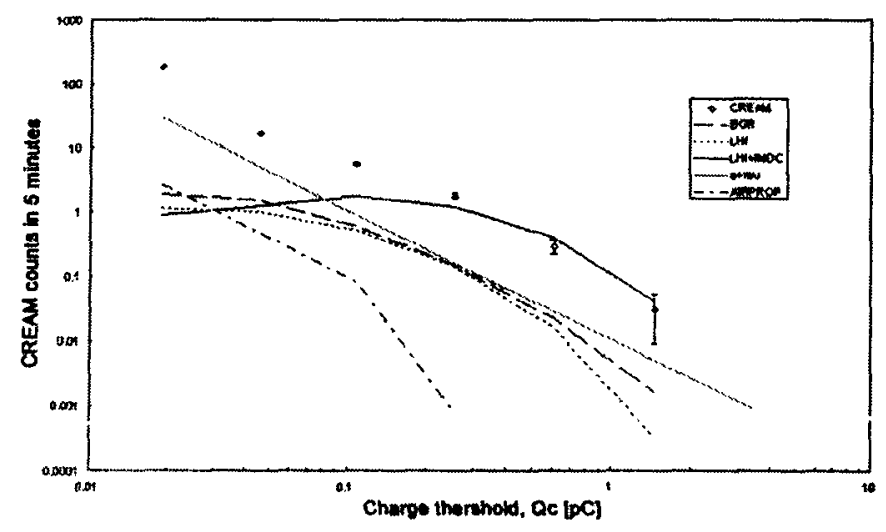

Fig. 20 - See cap. in Paper

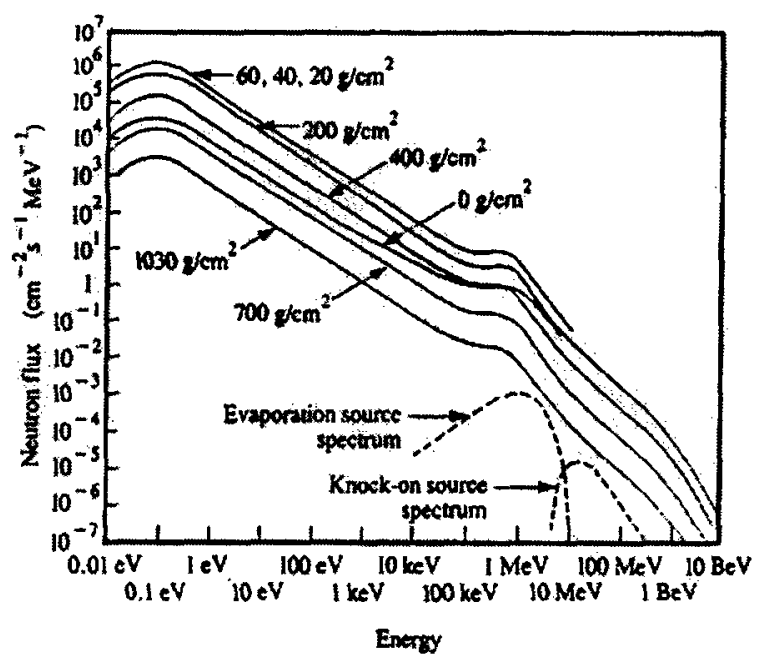

Fig. 21 - See cap. in Paper

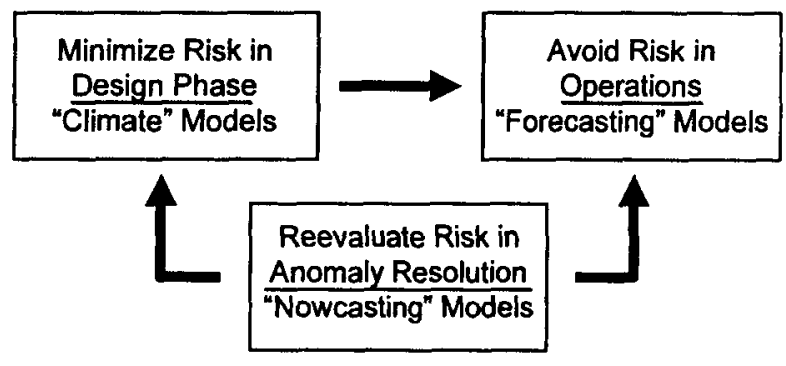

Fig. 22 - See cap. in Paper 